NOTICE WARNING CONCERNING COPYRIGHT RESTRICTIONS:

The copyright law of the United States (title 17, U.S. Code) governs the making of photocopies or other reproductions of copyrighted material. Any copying of this document without permission of its author may be prohibited by law. 


\title{
A VISUAL PROGRAMMING LANGUAGE FOR NOVICES
}

\author{
Technical Report AIP - 22 \\ Jeffrey G. Bonar \\ Computer Science Department and \\ Learning Research and Development Center \\ University of Pittsburgh \\ \& \\ Blaise W. Liffick \\ Department of Mathematics \& Computer Science \\ Millersville University
}

29 September 1987

This research was supported by the Computer Sciences Division, Office of Naval Research and DARPA under Contract Number N00014-86-K-0678. The Learning Research and Development Center AI Equipment and software base was developed under ONR Contract Nos. N00014-83-6-0148 and N00014-83-K-0655. The original version of Bridge was developed under Air Force Human Resources Laboratory Contract F41689-84-D-0002, order 0004. The opinions expressed do not necessarily reflect the position or policy of the agencies and no official endorsement should be inferred. Reproduction in whole or in part is permitted for purposes of the United States Government. Approved for public release; distribution unlimited.

BridgeTalk came out of a richly interdisciplinary research community of computer scientists, psychologists, programmers and educations, all concerned with improving the quality and impact of educational applications of computers. Discussions with Alan Lesgold and Lauren Resnick, helped to crystalize the ideas presented here. Mary Ann Quayle, Jamie Schultz, and John Corbett contributed to the design and implementation of early versions of BridgeTalk. Bob Cunningham contributed substantially to the design of later versions and was the principle programmer on the project. Paul Beatty, Vikki Pitts, and Perry Riggs also contributed to later versions. The Chips interface design tool was developed and implemented by Bob Cunningham and John Corbett. An earlier version of the discussion on novice programming language design appears in Technology and Learning, published by Lawrence Erlbaum Associates. 


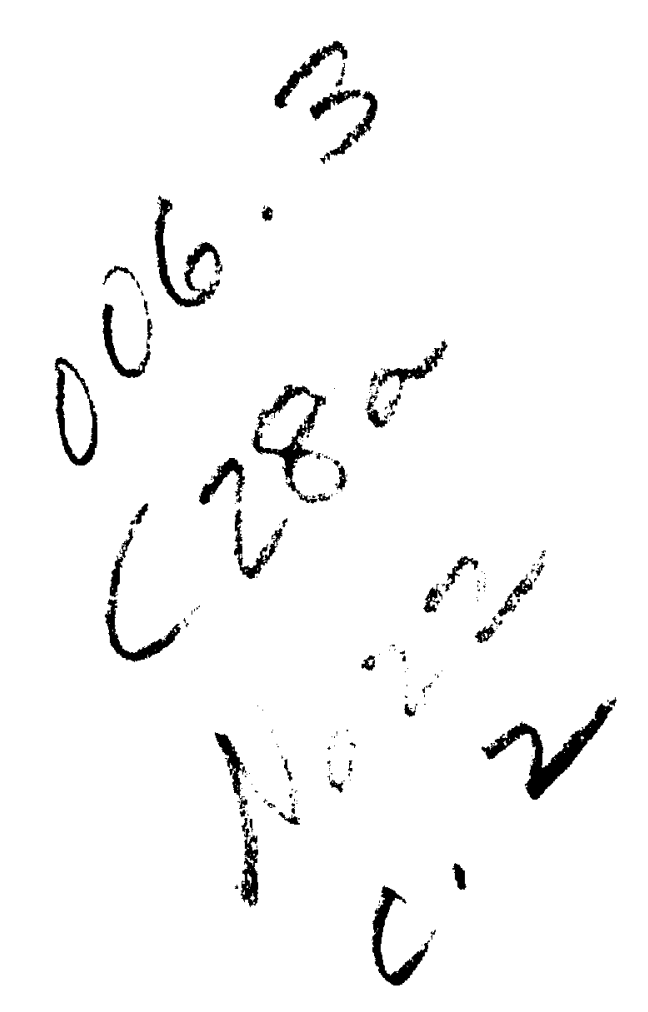


REPORT DOCUMENTATION PAGE

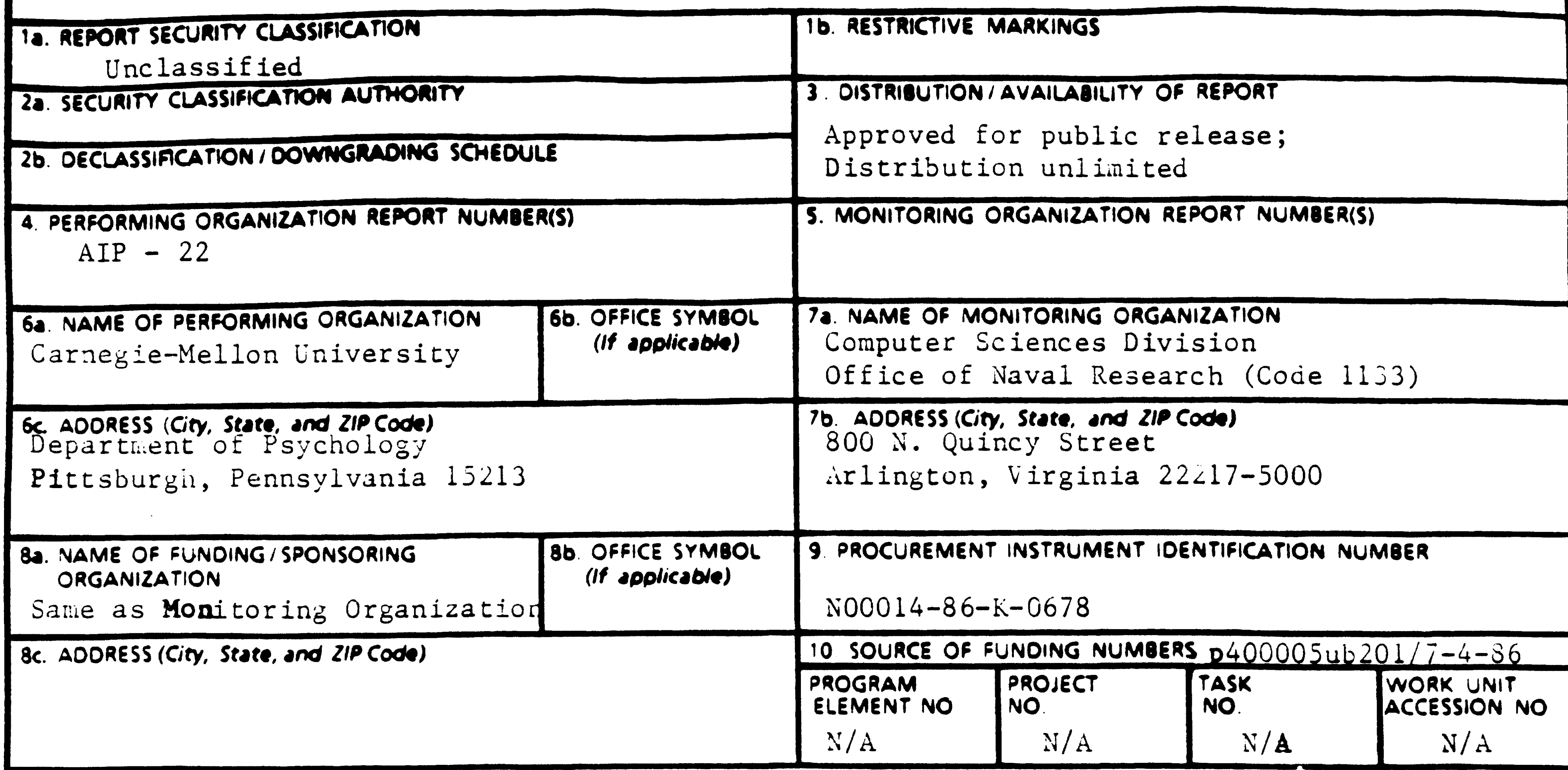

\section{TITLE (Include Security Classification) \\ A Visual Programming Language for Novices}

12 PEgSONAL AUTHOR(S) $\mathrm{B}$. Liffick

\begin{tabular}{|c|c|c|c|}
\hline $\begin{aligned} \text { 13a. TYPE OF REPORT } \\
\text { Hechnicai }\end{aligned}$ & $\begin{array}{l}\text { 130. TIME COVERED } \\
\text { FROM } 8 \text { SEPE } 1 \text { STO } 91 \text { Sept } 14\end{array}$ & $\begin{array}{l}\text { 14. OATE OF REPORT (Year, Month, OAY) } \\
87 \text { September } 29\end{array}$ & $\begin{array}{c}\text { 15. PAGE COUNT } \\
50\end{array}$ \\
\hline
\end{tabular}

iG SUPPLEMENTARY NOTATION

\begin{tabular}{|l|l|l|}
\hline \multicolumn{3}{|c|}{ COSATI COOES } \\
\hline FELO & GROUP & SUB-GROUP \\
\hline & & \\
\hline & & \\
\hline
\end{tabular}

18 SUBJECT TERMS (Continue on reverse if necessary and identify by block number) Teaching programming, visual programming language, programming languages

19 ABSTRACT (Continue on reverse if necessary and identify by block number)

We present BridgeTalk, a new approach to visual languages for novice programmers. The design of BridgeTalk is based on data about how novices learn to program. BridgeTalk allows novices to program with programming plans -- frame-like objects that capture essential program components like "keep a running total" and "iterate down a data structure". Novices are focused on the interactions between plans, not on the implementation details for a particular plan. Beginning with plans as a basis for a novice programming language, we were forced to develop a programming formalism that can deal with multiple levels of detail, merged plan implementations, and interrelationships between plans. The actual visual presentation for the language is based on six "design, implement, test with students, and redesign" cycles.

\begin{tabular}{|c|c|c|c|}
\hline $\begin{array}{l}\text { 20. DISTRIBUTION / AVAILABILITY OF ABSTRACT } \\
\square \text { UNCLASSIFIED/UNLIMITED G SAME AS RPT }\end{array}$ & $\square$ OTIC USERS & 21 ABSTRACT SECURITY CLASSIFICA & ION \\
\hline $\begin{array}{l}\text { 22a NAME OF RESPONSIBLE INDIVIDUAL } \\
\text { Ur. AIan L. Meyrowitz }\end{array}$ & & $\begin{array}{l}\text { 22b TELEPHONE (Include Area Code) } \\
(202) 696-4302\end{array}$ & $\begin{array}{l}\text { 22C. OFFICE SYMBOL } \\
\text { IY OOOIL }\end{array}$ \\
\hline
\end{tabular}



"But if we look at what a programmer would say about a program to a colleague who wanted to work on it or use it, very little of the description appears anywhere in the code."

Winograd [1979]

\section{Introduction}

There is a wealth of evidence that experienced programmers use a large set of programming plans: standard templates and structures for accomplishing typical programming tasks [Bonar and Soloway, 1985; Soloway and Ehrlich, 1984; Spohrer, 1985; Waters, 1985]. Example plans include "running total" and "iterate through a data structure looking for a distinguished value". Our objective is to take advantage of plans to provide novice programmers with an environment for learning to program. This environment begins with a high-level, plan based language, but permits and encourages the ultimate use of a standard language like Pascal. For several reasons the plan language has been designed as a visual language. This paper reports our initial efforts at formalizing and generalizing this plan language.

In learning a programming language, novices have two fundamental difficulties, both of which are addressed in our plan language:

Relating experience with informal plans to programming: Empirical evidence [Bonar and Soloway, 1985; Kahney and Eisenstadt, 1982] suggests that novice programmers bring a vocabulary of programming-like plans from everyday experience with procedural specifications of activities expressed in natural language. These plans come from experience with step-by-step instructions like "check all the student scores and give me an average" or "see that hallway, if any doors are open close them." These informal plans, however, are often extremely difficult for novices to reconcile with the much more formal plans used in standard programming languages. Note, for example, that both example phrases involve an iteration without any specific mention of a repeated action.

Translating formal plans into programming constructs: Even if a student recognizes particular formal plans, they are likely to have difficulty translating plans into programming code. A running total, for example, is implemented in Pascal with four statements, dispersed throughout a program: a variable declaration, an initialization above a loop, an update inside that loop, and a use below the loop. Epohrer et al. [1985] have shown that plan-to-code translation errors account for many student errors.

In responding to these problems, we have developed four objectives for our visual plan-based programming language for novices: 
(1) Allow users to "connect" to their informal (primitive) plans. Unless novices can recognize how their own understanding of plans fits into the programming environment they are being faced with, they will find it impossible to formulate correct solutions.

(2) Support novice programmers in learning a "vocabulary" of programming plans. Eventually, the user would begin to think in terms of the programming plans themselves, not the informal plans. Not only is there a catalog of plans, but students are able to create their own plans.

(3) Support novice programmers in learning how to implement plans with a standard programming language. The ultimate goal is that the user will learn enough to be able to program in a standard programming language such as Pascal.

(4) Support the use of plan-like composition of programs. Plans can be seen as the essence of good program comments. To the extent that a program uses a vocabulary of plans, the program will be easy to read and understand.

These goals create an environment that supports a new, higher level programming language, as suggested by Winograd [1979]. Though our intention is to teach a standard language, e.g. Pascal, not all users need continue on to this additional level of complexity. Those students that do not learn Pascal are limited to using the provided plans. The more a student learns about translating plans into code, the more directly they can express their intentions.

\section{Programming Languages for Novices}

The approach presented here grew from our concern with the conceptual distance between the syntax and semantics of programming languages like Pascal and the purpose and goals realized by that code. We felt that the programming task, as typically presented, confronts students with an enormous gap between goals and code. The BridgeTalk visual language presented here is intended to "bridge" this gap.

To illustrate the gap between goals and code in Pascal, consider the goal of "keeping a count" as implemented in Pascal:

- Above the loop - A programmer must declare a counter variable and initialize it to zero using an assignment statement.

- Inside the loop - The counter must be incremented, again using an assignment statement with a peculiarly non-algebraic construction:

Count : = Count +1 . 
Although an elementary concept, this is a very weird construction when viewed from outside the domain of programming. It requires a particular model of how computer memory is implemented in terms of extracting a value from a memory cell, incrementing the value, and storing the result back in the same cell. (Burstein [1986] discusses the assignment construct in detail.)

- Below the loop - The counter value must actually be used below the loop.

In summary, the simple goal of "keeping a count" has been spread throughout the program and buried in general purpose constructs. The design of these constructs is more closely related to the architecture of a register-based computer than to any problem actually being solved in the code.

Languages like Pascal are rooted in a programming model that is not closely related to the purpose and goals of the programmer using the language. Research into how novices learn programming confirms that the semantics of typical programming languages are not closely related to the way a typical novice understands a program [Bonar 1987]. Success with programming seems to be tied to a novice's ability to recognize general goals in the description of a task, and to translate those goals into actual program code (see, for example, Eisenstadt et al. [1981], Mayer [1979], or Soloway and Ehrlich [1984].) Our approach, embedded in the design of the BridgeTalk visual language, allows students to explicitly represent their goals and describe how those goals interrelate.

\subsection{Programming Plans: How Novices Learn to Program}

Most programming texts teach students almost nothing about standard programming practices between the statement level and the fairly vague "structured design" level. That there are standard concepts and techniques for implementing common tasks is rarely mentioned. Typical tasks like "keeping a running total," "iterating down a list," and "searching a binary tree" are usually only covered implicitly through examples. (See Bonar and Weil [1986] for a collection of such concepts and techniques for introductory Pascal programmers.) We call these "standard concepts and techniques" programming plans, after the usage introduced by the Programmer's Apprentice Project [Rich and Shrobe, 1976; Waters, 1981].

In the last few paragraphs, we discussed the difficulties novice programmers have mapping task goals into code. Programming plans provide a representation of the goals of their task, a set of tools for translating those goals into actual code. BridgeTalk is intended to provide students with a way to program by manipulating plans.

Programming textbooks typically introduce a programming language by discussing the syntax and semantics of each statement type. Unfortunately, this approach exacerbates a common novice tendency to adopt a syntactic matching strategy 
for problem solving. For example, physics students will often attempt to solve elementary mechanics problems by matching knowns and unknowns against standard formulae [Chi et al., 1981]. Their problem solving degenerates into a syntax-directed search with no understanding of the quantities being manipulated. Experts, in contrast, analyze a problem in terms of standard intermediate concepts and techniques from past experience. In physics, for example, these concepts and techniques include "component vectors", free body diagrams, and conservation of energy.

Programming novices exhibit syntactic strategies similar to those of the physics novices. In our video protocols of novice programmers [Bonar, 1985] we see novices working linearly through a program, choosing each statement based on syntactic features of the problem text or program code. Programming plans are exactly the concepts students need to step above the syntactic approach. Students can work on programming problems using standard approaches as encoded in plans.

A language that allows students to use programming plans has other advantages. Because the plans are high level, they allow the student to directly address interrelationships, in particular plan merging. Plan merging is necessary because programming plans typically have several facets that end up as dispersed lines of code. We saw this earlier with the counter plan example. When the counter increment must be placed inside of a loop body after the loop test, we are merging a facet of the counter plan with a facet of the loop plan. Errors in this plan merging process form a critical area of novice difficulty [Spohrer et al. [1985]. With an explicit plan representation, students can work directly with plans and plan interactions, without confusion about exactly how the merged plans will be turned into code.

\subsection{Why Current Programming Languages are Unsuitable for Novices}

The goals and constructs of standard programming languages are inconsistent with the needs of novice programmers. This can best be seen by examining the aesthetics used to judge standard programming languages: economy of expression, distrust of defaults and implicit behavior, emphasis on abstraction and abstraction tools, and efficiency on standard computer architectures. Here, we take up each point in detail.

\subsubsection{Economy of Expression}

Given similar functionality, programming languages with fewer constructs are almost always considered better then those with more. Pascal is considered an excellent programming language design because it managed to add substantial new functionality - user defined types - and embody a new approach - structured programming - in a language with substantially fewer constructs than its predecessor ALGOL 60. The history of LISP has been driven by language researchers looking for one abstract construct that can be used to efficiently implement a host of more 
specialized constructs. Finally, one of the most common criticisms of the language Ada is its large size and firm opposition to "subsets".

Economy of expression makes sense when considering the implementation of a programming language, but seems to interfere with an important part of learning programming. As discussed above, a wealth of evidence now exists that expert programmers use a large collection of programming plans - standard templates and structures for accomplishing typical programming tasks. Experienced programmers effortlessly implement hundreds or even thousands of such plans [Waters, 1985] in programming languages with relatively few - on the order of 50 - constructs.

Novice programmers, on the other hand, have major difficulties implementing plans with programming language constructs. Evidence from our own work [Bonar and Soloway, 1985; Bonar et al., 1986] suggests that novice programmers bring a vocabulary of programming-like plans from experience with procedural specification in natural language. The translation of these natural language plans into the the narrow, low level constructs provided by a programming language is a critical and often insurmountable problem.

Acquiring the new plans that are essential to mastering programming is certainly difficult. This difficulty is compounded because novices not only need to acquire the plans, but they also need to learn how to translate the plans into a programming language. Because the natural language plans are rooted in real world day-to-day procedural specifications, it is very tough for a novice to translate them into a sparce and formal set of programming language constructs.

To illustrate the general problem, consider the following excerpt from an interview with an introductory Pascal student. She was writing a program that reads, sums, and counts inside a loop. She had written the following lines (line numbers are given for reference):

\section{repeat}

$$
\begin{aligned}
& \text { Read (New); } \\
& \text { Sum := Sum + New: } \\
& \text { Count : = Count }+1 \\
& \text { until Count }>100
\end{aligned}
$$

The first author asked this student if line (3) was a "different kind of statements" than line (4). To our surprise, she stated that even though these statements "look alike," they were quite dissimilar. She went on to explain that line (3) "has something to do with something you are gonna ... take out of the loop with you." On the other hand, she explained, line (4) "keeps the loop under control." We contend the student has a deep understanding of the programming issues involved, even though she may have a weak 
understanding of the specific Pascal constructs. While she has a firm grasp on the plans to be accomplished, she has a much weaker grasp on the language design constraint that would cause statements with such different purposes to have such a similar look.

We conclude that the emphasis on economy of expression in most modern programming languages is misplaced in designing languages for novice programmers. By looking for ever more abstracted ways to express similar procedural behavior, modern languages have excised most clues of goal and purpose that are essential to novice understanding of a program. The student discussed above would have much preferred a language with two constructs, "counter update" and "running total update", to Pascal which subsumes these (and many other) plans in the assignment statement. For this reason, BridgeTalk is a richer language than standard languages like Pascal. The richness in BridgeTalk expresses a catalog of standard plans.

\subsubsection{Distrust of Defaults and Implicit Behavior}

Another trend in the development of programming languages has been the systematic removal of defaults and implicit behavior. Whereas early languages such as FORTRAN and BASIC assumed properties and initial values for variables - for instance, based on the first letter of the variable's name - such assumptions are rare in modern languages. The trend to deemphasize default and implicit behavior is consistent with the trend toward economy of expression. Typically, defaults and implicit behavior add more complexity to a language. For example, a programming language designer would argue against a default value mechanism when standard assignment statements at the top of a program can accomplish the same end. Even where defaults reduce the number of tokens needed in a program - implicit typing of variables, for example - the defaults complicate a formal description of how the program runs.

As with economy of expression, the lack of defaults and implicit behavior makes standard programming languages much more difficult for novice programmers. While it is no extra work for an expert programmer to know to explicitly specify that a counter is to be incremented by one, it is a confusing burden to a novice. As expert programmers, we all can remember (or conjure up) a few circumstances where a problem is best solved by incrementing a counter by other than one. Even though such circumstances are exceedingly rare, we force every novice to explicitly specify this, as if a counter were commonly implemented in some other way.

Consider another example. Plans like "iterate down a list" are typically implemented with constructs that begin by testing for a condition that indicates we have finished the entire list. Readers with programming experience will need to consider for a moment to realize that this is very peculiar. First of all, it makes little sense to put an ending test at the beginning of the construct, before we've talked about 
what the construct is to do. Second, it is strange that the list structure itself does not "know" where its end is, recognize the end, and take some appropriate default action to stop the iteration. Such examples of missing defaults are pervasive. We give novice programmers considerable extra work by forcing them to translate common plans into much lower level programming language constructs.

\subsubsection{Emphasize Abstraction and Abstraction Tools.}

Modern programming languages emphasize tools for abstraction. Current programming language design and development focuses on developing language constructs that capture and summarize structure and function for later use. For example, older languages provide control structures while more modern languages provide tools for interprocess communication, with the original control structures available as special cases. As another example, older languages focus on memory management while newer languages focus on data structuring and reuse of components.

As we have discussed above, non-programmers do not need tools for more abstract expression of programming plans; they need more direct ways to express the actual plans. The formal power of highly abstract constructs is in contrast to the pragmatic familiarity of programming plans. From the point of view of a procedural mechanism, an assignment statement is an abstraction that simplifies and subsumes a host of specific "changes to a variable," including, in particular, incrementing a counter. However, by stripping out the real world pragmatics that underlie programming plans, such abstractions make much of the programming process implicit and unavailable to novice programmers.

\subsubsection{Efficiency on Standard Computer Architectures}

A crucial constraint in most modern programming languages is that the language run efficiently on standard computer architectures. Pascal, for example, in its implementation of sets and rigid specification of ordering among program components, was intentionally tailored for efficiency of implementation. The designer of Pascal set the development of "implementations ... which are both reliable and efficient on presently available computers" [Wirth, 1971] as his second of two principal aims for the language. While such an aim is obvious in a language for professional programmers, it is questionable in a language for novice programmers.

Many programming constructs are quite confusing to a novice programmer. For example, the assignment statement has peculiar semantics when seen from a perspective other than one that understands the basic working of registers and synchronous busses. In our daily experience it is rare that copying is simpler to specify than movement, as it is with assignment. Furthermore, in our daily experience we 
rarely encounter values that are easier to destroy than to displace, again as it is with assignment.

\subsection{How Can Visual Languages Help Novices}

There are two basic reasons to turn to a visual language in a programming language for novices. First, a visual language provides the flexibility and expressiveness needed for a novice language to express a large vocabulary of programming plans. In our experience, a linear textual version of a plan-based programming language is quite unwieldy. Second, from a point of view of novice cognitive capability, a visual language is less likely to tax a novice's working memory. We discuss each of these points in detail.

\subsubsection{Visual Languages for Expressing Plans}

In BridgeTalk the emphasis is on identifying the formal plan components and expressing their interrelationships. As discussed above, correct expression of plan interrelationships has been shown to be critically difficult for novices, particularly where those interrelationships result in the merging of several plans into one sequence of code [Spohrer and Soloway, 1985]. BridgeTalk provides an environment where students can focus on plan interrelationships.

The various BridgeTalk constructs are implemented as icons that can be picked up, manipulated, placed, and connected together. The critical constraint that dictated this approach was the need for a language that allowed atomic plans with multiple connections of different types. The essentially linear nature of textual languages makes it very difficult to express interconnections. Furthermore, since plans represent rich, high-level programming objects, it is sensible to depict them as icons that suggest their function. In fact, the current iconic representation was suggested by the confusion of students using earlier textual versions. (See section 4 where we discuss the formative evaluation of BridgeTalk.) Although the plans of BridgeTalk are not completely general, they do allow a student to focus on the pragmatic knowledge that stands above particular programming language implementations of that knowledge.

\subsubsection{Visual Languages and Novice Cognition}

Anderson [1985] has documented novice programmer errors which arise from overloading the student's working memory. The difference between novices and experts, they found, was the ability of experts to organize and structure their knowledge within working memory. Other work (cited earlier) points to plans as the kinds of structures used by experts. By providing plan icons and a way to interconnect those plans, we give students a structure to use in organizing their understanding and problem solving. 


\subsection{Design Goals For BridgeTalk}

There are four objectives for BridgeTalk:

(1) Allow users to "connect" to their informal (primitive) plans.

(2) Support novice programmers in learning a "vocabulary" of programming plans.

(3) Support novice programmers in learning how to implement plans with a standard programming language.

(4) Support the use of plan-like composition of programs.

We take up each of these goals separately.

\subsubsection{Connecting to Informal Plans}

Unless novices can recognize how their own understanding of plans fits into the programming environment they face, they will find it impossible to formulate correct solutions. Novice programmers have a large vocabulary of informal plans based on experience with step-by-step procedures in everyday life. We want BridgeTalk to connect to and build on this understanding. A subset of the plan icons in BridgeTalk must connect to the collection of naive plans brought in by programming novices.

This goal yields several benefits. First, there is the obvious benefit that students have at least some familiarity with the system, even before their first use. Furthermore, the informal plans brought in by a novice are typically a simplified form of plans that are central for experienced programmers. Finally, a simplified novice plan forms a good starting place for understanding the related set of richer, more formally specified expert plans.

Note that we are not arguing that a novices' collection of naive plans is sufficient for sophisticated programming. Instead, we are recognizing the importance of giving a beginner an anchor point in a new domain. We are very clear that experienced programmers have a much richer set of programming plans than a novice. We are also clear that the plans of an expert programmer are more fully articulated and elaborated than the plans of a novice. Nonetheless, we see great value in supporting the growth of a novice from primitive plans that are already known.

\subsubsection{Developing an Explicit Vocabulary of Programming Plans}

Although we begin with a student's informal plans, our ultimate goal is that a student leaves a programming course with a rich set of highly articulated programming plans. Even in a language with no explicit plan structure, e.g. Pascal, successful students do leave a course with a rich vocabulary of plans. With BridgeTalk, we provide a mechanism for this vocabulary to be an explicit part of programming instruction. 
An explicit vocabulary of plans provides an opportunity to formally specify programming curriculum. Although elementary programming courses often discuss issues of design, abstraction, and structure, this is rarely done with any rigor. We suggest that the rigor is missing because there is no adequate vocabulary with which to discuss programming design. Typical programming constructs operate at too low a level. At the other end of the spectrum, formal approaches to correctness offer little to a student still attempting to understand the design space of programming.

Essential to the development of a plan vocabulary are techniques for extending and modifying plans in a systematic way. In BridgeTalk we provide techniques for extending and specializing a particular plan. Students just beginning with BridgeTalk can use the plans provided with the system. More experienced students can develop their own plan vocabulary.

\subsubsection{Implementing Plans With a Standard Programming Language}

If a student desires, BridgeTalk should support that student in learning to program in a standard programming language such as Pascal. In general, this means that the plan formalism must provide a way to derive a conventional program from any plan program. BridgeTalk provides a mechanism whereby students can successively "look inside" a plan, and at each level reveal an implementation closer and closer to typical programming constructs. This approach takes advantage of the strengths of both plans and programming constructs. On one hand, the plans abstract away from particular programming constructs, specifying higher level goals. On the other hand, the programming constructs are more general purpose than the plans.

The system is layered for interrelated implementation and pedagogical reasons. By providing various layers, we can take advantage of shared structure between various plans. For example, the counter plan is a specialization of the running total plan, and is implemented that way. Pedagogically, it is valuable for students to see the shared structure inherent in the various plans. By using multiple layers, we need only show the plan components required to illustrate the commonality, without showing all the underlying detail. So, for example, the counter plan can be described as a specialization of the running total plan where the update is always one. Note that most details of the plan implementations need not be understood to understand their commonality.

\subsubsection{Supporting Plan-like Composition of Programs}

The final design goal is that BridgeTalk support programming by plan composition. To support this goal each particular plan has a single icon. We feel this is crucial if we are to allow a student to focus directly on plan interconnection separate from other issues. As discussed above, one of the crucial difficulties of a programming language like Pascal is that simple plans can end up expressed in several dispersed 
lines of code. Our goal for BridgeTalk is a representation that maintains a plan as an atomic element.

Even though the plans are atomic, we must be able to distinguish between components contained within the plan. For example, a looping plan needs to have separate subcomponents that represent a test, a body, and a modification that can change the value of the test.

\section{A Visual Language for Novices}

\subsection{Informal Overview}

A key design constraint of the BridgeTalk language is that each particular plan has a single icon. We feel this is crucial if we are to allow a student to focus directly on plan interconnection separate from other issues. As discussed above, one of the crucial difficulties of a programming language such as Pascal is that simple plans can end up expressed in several dispersed lines of code. Our goal for BridgeTalk is a representation that maintains a plan as an atomic element.

Consider the following example programming problem, which is used in our discussion. We call this the Ending Value Averaging Problem. The problem is:

Write a program which repeatedly reads in integers until it reads in the integer 99999. After seeing 99999, it should print out the CORRECT AVERAGE without counting the final 99999.

The BridgeTalk plan icons used for the Ending Value Averaging Loop plan are shown in Figures 1 and 2. The general metaphor of the plan language is that of puzzle pieces being fit together. Plans with similar shapes have similar kinds of roles in a program. Plans that express a sequence of values in a variable, called "variable plans," are shown as squares (see Figure 1). Each of these plan icons embodies the entire semantics of a particular plan.

The Counter plan, for example, keeps and shows a value (the count so far), knows to initialize itself to zero, and increments its value by a specified amount every time control flows through it. The Running Total plan similarly keeps and shows a value (the total so far), knows to initialize itself to zero, and adds the value of another plan into the total so far (how the connection with the plan that supplies the value is expressed is discussed below.) The Input plan gets a sequence of values from the user of the program. Every time control passes through the plan a new value is requested from the user.

In addition to the variable plans, Figure 1 shows a Compute plan. The Compute plan uses values supplied by variable plans (the connection to a variable plan is 
described below), and an operation selected by the user. The operation is specified by mouse selection on the operator box and selecting an operator symbol off a pop-up menu. When control passes through the Compute plan the operation is performed with the current values of the associated variable plans.

Figure 2 shows a New Value Controlled Loop plan, one of the most complex plan icons in BridgeTalk. There are four components of the New Value Controlled Loop plan:

new value generator - A variable plan to produce a series of values, each of which is tested to determine when to exit the loop. Typically the new value generator is an input plan that requests values from the user. It could also be a random number generator or a traversal of a data structure.

end of loop condition - A test to determine when to exit the loop. Note that this test is constrained to do a particular kind of test: checking each new value. Another loop that requires a different sort of test - for example, to see if the running total has exceeded a particular value requires a different plan.

body of loop - A series of plan icons to be executed in the body of the loop.

actions to be performed after loop is complete - A series of plan icons to be executed when the loop completes.

The key idea with the New Value Controlled Loop plan is to hide all the syntactic and control flow complexity that a student would need to confront to implement such a loop in a standard language. (Soloway, et al. [1983] presents detailed data on this complexity.)

Figure 3 shows a BridgeTalk solution to the Ending Value Averaging problem. The focus of BridgeTalk is on the connections between the plans. In particular, there are two kinds of connections that students must master: control flow and data flow. Control flow expresses the order of execution for the plans. In BridgeTalk control flow is expressed explicitly by connecting the puzzle-piece tabs together. Plans are executed in a top-to-bottom order. So, for example, in Figure 3 the Compute Plan is executed before the Output Plan.

In addition to control flow, students must also express data flow. Specifically, students must show how values computed in one plan are used in other plans. This is done with a special plan called a Value plan. Value plans can be placed in holes within plans that are expecting values from another plan (a Value plan and corresponding hole are shown in Figure 4.) Value plans are created by selecting the box labeled "Value" within a plan that can produce a value. Figure 5a shows a Running Total plan whose value box has just been selected. Such a selection creates a Value plan icon that is 
attached to the mouse cursor. The data flow connection is established by dragging the Value plan to the plan that needs the value, and placing it in the appropriate hole. Figure $5 \mathrm{~b}$ shows the Value plan from the Running Total plan being placed in a hole of the Compute plan.

We have made the decision that BridgeTalk expresses control flow in a more direct way than data flow. Control flow connections have a direct visual expression. If plan $A$ is executed before plan B, the tab on the top of B's icon fits into the slot on the bottom of A's icon. Data flow connections, while specified directly by dragging an object from one plan to another, leave a much more subtle visual clue. In theory, we could have designed the plan language to emphasize visual expression of data flow instead of control flow, or even to emphasize either, depending on a mode selected by the user. One earlier plan language had a visual representation for both control and data flow simultaneously, but proved to be too complex and unwieldy for our students. We chose to favor control flow over data flow in order to best match Pascal, the current "default" novice programming language.

\subsection{Formal Description}

This section describes a formal representation for plans that forms the basis for our visual programming environment to aid novices in learning to program. Our approach allows several representations of a problem solution. The user is able to navigate from one representation to another in order to obtain different points of view on the solution. The problem solution can be modified from any one of these representations, or levels. The solution is descriptive at the highest level, prescriptive at the lowest. This top level uses icons to identify specific plans that the user links together appropriately. At the lowest level is source code for a textual, procedural language, such as Pascal.

\subsubsection{Plan Representation}

The plan formalism is based on object-oriented programming. For each type of plan there is a class that specifies the local data and operations of that plan. Instances of a plan class can then be created, each with their own copy of the local data. The user organizes these instances into a particular execution order using abuttment, embedding, and merging.

Each plan is represented with up to four parts:

ParentClass - this section provides a link indicating a hierarchical relationship among plans for purposes of taxonomy and inheritance. Each plan is a member of exactly one class. If this section is missing, there is no parent class to the plan. 
Slots - each plan can have zero or more slots, which provide one of two types of entities - data or plan links. The data slots are untyped at this stage of the system, and contain a single value. One slot can be distinguished as the "value" slot for this plan, meaning that the plan acts like a function and creates a single value that can be used elsewhere. The plan links provide a method for referring to other plans for purposes of abuttment and embedding.

Initialization - this optional section contains "executable code" that is performed once when the plan is first accessed. Whenever a plan is entered, if its initialization section has not been previously executed, it is fired. As a special condition, when a loop plan is entered, it fires its initialization section and the initialization sections of all plans contained in its body.

Execution - this optional section contains "executable code" that is performed whenever the plan is accessed.

The code given in the Initialization and Execution sections is expressed in a simple pseudo-code which creates the required control structures. This code can be easily translated into a standard programming language such as Pascal, given the plan representations and the pseudo-code. There are sufficient constraints on the representation to make this a straightforward process. It can be done incrementally, allowing for user modification of the code. The user will have access to this code, allowing him/her to examine the way a plan is implemented in a standard programming language.

Figure 6 gives an example of the above scheme. This example forms a hierarchy of refinement for the notion of a counter. At the top-most level is a Loop Action plan. The lack of a ParentClass designation simply means that this plan inherits from no other class. In addition, there are no Slots, Initialization, or Execution sections associated with this type of plan. Its purpose in the taxonomy of plans is to indicate that any plan decended from the Loop Action class must be used within the body of a loop.

Next, the Running Total plan contains three slots, all data slots. The angled brackets $(<>)$ indicate data that has no pre-specified value. The slot Total has been prefixed with "value." as an indication that it is the distinguished slot that will be taken as the value of this plan. Finally, the Initial slot has been given an explicit value of zero. The asterisk indicates that this is a default value, and can therefore be changed by the programmer. 
The Initialization and Execution sections give typical "code" for how a running total works. As a member of the Loop Action class, the Running Total plan will naturally be embedded in a loop of some kind, so there is no need to explicitly specify the loop itself here.

The Constant Running Total plan is a specialization of the Running Total plan. The only difference is that the addend is a constant of some sort, so that this plan sums a single value over and over. The one slot in this plan shows two additional notations. First, the slot Addend that was used in the Running Total plan has been renamed to Increment. This renaming is indicated syntactically by using a concatenation of the two names. The renaming makes it easier to construct meaningful messages for the user. Second, this slot has been redefined to include a link to another plan, namely the Constant plan which asks the user for a value for the constant to be summed.

Finally, the Counter plan shows a further refinement of the Addend.Increment slot to be a specific value of 1 (default). In addition, the slot Total that was inherited from some parent class (in this case, from Running Total) has been renamed to Count. The renaming does not affect the designation of this slot as the "value" slot, however.

\subsubsection{Inheritance}

Each plan must specify a parent class explicitly, unless there is no parent. This provides a linkage for the inheritance mechanism.

Slots are inherited from all predecessors, but may be renamed or redefined. Note in the example given in Figure 6 that the Counter plan has inherited (and renamed) the Total slot from the Running Total plan, although the intervening plan (Constant Running Total) does not explicitly reference it. In addition, the Counter plan inherits Total's designation as the distinguished value of the Running Total plan.

The Initialization and Execution sections can also be inherited. In this case, both are inherited by successors from the Running Total plan. The renaming and redefining mechanisms allow us to think of the execution of the Counter plan as

Count $<--$ Count +1.

In addition, it allows us in conversation with the user to describe the " 1 " as an "increment" rather than as an "addend."

\subsubsection{A More Complex Example}

Figure 7 shows the representation of the Ending Value Averaging Loop plan and its components. This plan is used to calculate the average of a running total of values that are entered by the user. 
Some additional notation occurs in this example. Note that it is possible to pass values to linked plans. For instance, in the case of the Average slot, the Average plan is passed the values of Total and Count with which the Average plan will compute the average. These values are substituted for the slots Dividend and Divisor, respectively, in the Average plan. The Output slot sends this average to the Output plan.

The Execution section of the Ending Value Averaging Loop plan shows how slots can be executed by sending them an Execute message. The meaning of this code is that the slots identified are to be executed sequentially, forming an abuttment of the plans contained in the named slots.

Note that the Loop slot contains a reference to the Sentinel Loop plan. This Sentinel Loop plan is a subclass of the New Value Controlled Loop plan. The Sentinel Loop plan does not have any executable sections, but does define three new slots that will be used during execution. In addition, even though the Sentinel Loop plan itself does not contain a slot called Body, as referenced in the Loop slot of the Ending Value Averaging Loop plan, it inherits such a slot from its parent class, the New Value Controlled Loop plan.

The Body slot requires some additional explanation. Generally, a slot being used as a parameter contains the name of a single value or slot. In the case of Body, however, several slots (and, therefore, plans) can be abutted to form, for instance, the body of a loop. The Body slot, then, acts similar to a BEGIN-END block in Pascal. In this example, the body of the Ending Value Averaging Loop plan is identified as being the Total slot (and, consequently, the Running Total plan), followed by the Count slot (linking to the Counter plan.

Finally, note that the code for performing the actual looping action appears only in the New Value Controlled Loop plan. The Ending Value Averaging Loop plan and the Sentinel Loop plan leave this detail to another level so that it can been hidden from the user to some extent. In this case, this executable code is inherited by the Sentinel Loop plan, where the values given in its slots (which are references to other plans) are used to "fill in the blanks" of the code. In this way, a subclass plan can redefine slots that have been originally defined in its parent plan.

The Body of the loop in the executable code given in the New Value Controlled Loop plan actually comes from the Ending Value Averaging Loop plan. In this way, the actual contents of the loop body can be customized to fit the requirements of particular plans. In this case, it is the Ending Value Averaging Loop plan that should define that the body of the loop contains Running Total and Counter plans. The Sentinel Loop plan is not responsible for this detail, since its only concern should be how to construct a sentinel-valued loop. 


\subsubsection{Meeting the Goals}

The notions of programming plans and informal natural language plans are fundamental to this system. The "connection" between these two types of plans obviously must be provided in a natural, easily understood manner in order to be obvious to a novice. Using plans already is a first step toward providing an intuitive environment. It will be the responsibility of the interface to provide the rest of the connection that is required. This is one of the main reasons why a visual programming environment is desireable. The interface would naturally need to include appropriate icons for the available plans, a graphics editor for creating a program, execution displays, etc. The rest of the discussion in this section assumes a suitable interactive interface, much like the one currently available in the Bridge programming tutor [Bonar et al. 1987].

Initially the novice would use plans at the highest possible level, constructing programs by selecting from a menu of plan icons and connecting the icons together using abuttment, embedding, and merging. For instance, he/she might use the Ending Value Averaging Loop plan with only the understanding that such a plan. would somehow total up a series of numbers and compute their average. This can be seen simply by looking at the representation for Ending Value Averaging Loop plan given in Figure 7, for instance, without referring to any of its sub-plans. Exactly how this will be presented to the user visually is not restricted by the representation given above. The actual formal representation could be one of the alternate representations available to the user, or could be just the base representation for all others without being visible itself.

A curious student, however, would probably want to know more about the plans identified in the slots of the Ending Value Averaging Loop plan. In such a case, the user could ask the system to navigate the hierarchy so that he/she could view, for instance, the Sentinel Loop plan. In this way the user can study the taxonomy of the plans, and learn something about plan construction.

Further interest might lead the user to examine the code that implements the Sentinel Loop plan (as inherited from the New Value Controlled Loop plan). This can be derived from the representation given in Figure 7. Exactly what form this will take is yet to be determined. Possibilities include diplaying the pseudo-code shown in Figure 7 , as well as an implementation in a standard language such as Pascal. In addition, it is possible to allow the user to modify this code directly, rather than relying entirely on the iconic representations of the plans. This lets the user -- as his/her skill grows -- to define his/her own plans. Selective execution of code is also possible with the system. This all helps the user to understand programming plans and their peculiar vocabulary. 
The navigation described above takes place in two distinctly different ways. In moving from the Ending Value Averaging Loop plan to viewing the Sentinel Loop plan, the user is scanning the details of the plan itself and its various component parts. Figure 8 shows a graphical representation of the Ending Value Averaging Loop plan and its components, as developed from the plan description of Figure 7 with all inheritences resolved. This figure has fairly standard connotations of hierarchy of the plans involved. It also contains some indication of plan construction -- the multi-branched lines indicate abuttment of plans, as in the case of the Loop, Average, and Output slots of the Ending Value Averaging Loop plan, and the Running Total and Counter plans (via the Total and Count slots, respectively) contained in the Body of the Sentinel Loop. This figure shows the plans involved with the Ending Value Averaging Loop mechanism at their highest level, which is essentially descriptive.

A second way that the plans might be navigated, changing to a different point of view, is through the Class hierarchy. This was somewhat implied by viewing the code implementing the Sentinel Loop plan above, inherited from the New Value Controlled Loop plan. Figure 9 shows a more specific example. Counter is shown to be a sub-class of the Constant Running Total plan, which in turn is a sub-class of the Running Total plan, which is a Loop Action plan. The slots of the Running Total plan are inherited by the lower levels. In this case, the Initial slot is inherited without modification by lower levels.

The Constant Running Total plan is shown redefining and renaming the Addend slot from its predecessor. The Counter plan also redefines this slot further. The Counter plan also renames the Total slot from above.

Navigating this hierarchy is considerably different than in the previous case shown in Figure 8. When moving from the Counter plan to the Constant Running Total plan, to the Running Total plan, we are moving from more specific levels to more general ones. It is more specific to say that we are counting, with an increment of 1 , than to keep a running total of some unspecified addend.

This hierarchy can be considered as orthogonal to the one shown in Figure 8. It represents additional detail that the user can explore for a deeper understanding of programming. Traversing these levels also lets users explore the plan taxonomy on

another level. In this way the user gains a more sophisticated point of view of the programming environment.

\subsection{Implementation}

A detailed discussion of the implementation of the plan language discussed above is beyond the scope of this article. The current prototype is implemented in LOOPS, an 
object-oriented extension to Interlisp-D on Xerox $11 x x$ workstations. BridgeTalk is embedded within the Bridge intelligent tutoring system for teaching Pascal [Bonar, et al. 1987]. The visual language is developed in Chips, a tool for building direct manipulation interfaces developed at the University of Pittsburgh's Learning Research and Development Center [Corbett and Cunningham, 1987]. Chips was particularly valuable in allowing the rapid development of prototype visual interfaces for testing with students.

BridgeTalk is the result of an indepth cycle of research, design, testing with students, and evaluation. As is documented in detail in the next section, BridgeTalk has been through six distinct generations of that cycle. The first two generations each took six person-months of program development time, along with one person-month each of empirical testing. The last four generations were each developed in less than three person-weeks, using the Chips interface design tool. Each of these last four generations also took about a person-month of empirical testing.

\section{Formative Evaluation and Design History}

BridgeTalk has not been systematically tested against a standard programming language like Pascal in a classroom situation, though such a test is planned. BridgeTalk is the result, however, of a long process of formative evaluation and development. BridgeTalk has been extensively tested with students through a number of different versions. In this section we summarize the design history of BridgeTalk over the course of 6 generations.

\subsection{Generation 1}

Figure 10 shows an Ending Value Averaging solution in the original version of BridgeTalk. The student uses a mouse to "pick up" and place each element in its proper place in the developing program.

There were two main objectives in this initial version of BridgeTalk. First, the student was to gain a better understanding of the flow of control implied by the various plans, and the interrelationships of the various plans. In the case of the loop construct, an icon was supplied that was meant to show graphically what the looping entailed. Two other icons, for input and output, were also supplied in an attempt to illustrate their function.

The second objective was to begin to show a separation of the various roles each plan may have. For instance, in the language of Figure 10 the user is faced with the need to identify where the initialization of a variable should occur. In the case of the Counter Variable plan, note the initialization and increment roles in separate locations in the figure. 
This version of BridgeTalk was deemed unsatisfactory for several reasons. First, the user was required to develop an entirely new vocabulary in terms of the specific plan phrases given. The phrases used were somewhat cryptic, making identification of the proper plan difficult.

A second problem was with the icons. Aside from their crudity, they were not particularly indicative of the function represented. The use of icons was also limited to only three constructs, input, output, and looping. In addition, those icons served no dynamic function, but were only somewhat abstract graphics used to highlight the particular plans. The main reason these specific three plans were chosen for graphical representations is that they seemed easily represented. It simply was not clear how to represent the other plans neatly using this sort of graphics.

A third problem was that this new representation of a problem solution was still static. This meant that the user still had to imagine how this solution actually would work. The static nature of the solution representations required too much computing skill from the user.

\subsection{Generation 2}

Version 2 of BridgeTalk was more data oriented. More complete descriptions of the various roles of each plan were given. Figure 11 shows a selections of plans from this second version. Plans are represented by boxes containing slots to be filled. Each slot represents a role, represented as a tile, of some other plan. This helped make each plan more self-explanatory. The underlying notion was that a plan could be more systematically treated as a frame with slots. The control structures became frames, and data were slots.

Figure 12 shows how a solution to the Ending Value Averaging problem was formed. Either an entire plan or just its slots could be used in the final solution. In this example, the Control Loop with Sentinel plan is used in its entirety. Its open slots are filled with tiles from other plans. In the case of the Count How Many plan, only its tiles are used, not the entire box identifying the plan.

While this approach does indeed make the data objects being manipulated more identifiable, and does give more explicit help to the user in terms of explaining the plans, there are still many problems. To begin with, the layout employed implies a fairly loose interpretation of plans by the user. In some cases, the entire plan's box is used. In others, only the tiles are used. In addition, sometimes these tiles are used to fill in slots of other plans, while at other times they are simply placed individually in the figure, with no direct connection to any other object in the figure. How does a novice interpret these differences of use? 
This approach also was too textual, giving up any advantage that might be gained from a more graphical representation. While the use of graphics in the previous version of was not particularly innovative, there at least was some suggestion of the dynamic flow of control.

Finally, this version did not account for the prospect of nested plans. How might the Compute the Result plan be placed inside the Control Loop with Sentinel plan, for instance?

\subsection{Generation 3}

The next version added more suggestive shape to the representation. In addition, it attempted to deal with the nesting issue. Figure 13 shows a loop with other plans following it. Preceding the loop plan are representations of initialization roles for two different variable plans. The basic representation is still, however, based on the notion of frames and slots.

Although this version adds some graphics, as in the original version the graphics are not particularly intuitive. In addition, they are still static. Up to this point in the development, however, it was not clear that an executable version of this phase was desireable. After lengthy consideration, it was decided to approach the phase with an iconic language that was executable.

\subsection{Generation 4}

This is the first entirely graphical representation of BridgeTalk. Each plan is represented by its own graphical object. The focus of this representation was on the merging and coordination of the plans, for instance as in the case of the actions to be performed as the body of a loop. In addition, this version was executable, allowing the user to see the flow of control as well as changes in data.

Figure 14 shows an object representing a generalized Loop plan and a Compute plan. In this case, the Loop plan currently contains a Counter plan and a Running Total plan. The Loop Test is on a slide mechanism, and the user must position the test with respect to other actions to be taken inside the loop. The Increment by and Update by roles of the plans embedded in the loop are also on slides. The position of the Initialize role of each of these plans is designed to indicate that they occur before the loop begins.

The Value roles hold the current value of each of the plans. The boxes containing these values are given unique shading to make them distinguishable throughout the program being created, as in the value implied by the Input plan given in the Loop plan. These values can be copied to other locations in the program.

In Figure 15 you can see how these mechanisms work in a typical example. In this example, the test condition of the loop has been positioned to occur before the Update 
and Increment portions of the plans embedded in the loop. Also note that the value generated by the Input plan is the same value used by the Running Total plan in its Update by role (the shading of the two boxes is the same). Also, the Value role of the Running Total is used in the Compute plan, along with the Value from the Counter plan to produce the final Output of the program.

Note that an explicit flow control line has been attached between the Loop plan and the Compute plan, and between the Compute plan and the Output plan. Also, there is a flow line on the side of the Loop plan. These lines are animated as the program is executed to indicate the order of execution. In the example shown in Figure 15, three numbers were entered, producing a total of 120 . The average was computed as 40 .

While this version has the virtue of the suggestiveness of its icons, and it is dynamic in nature, there is still at least one major problem that it creates, namely the inconsistent semantics of its graphical constructs. For instance, control flow is shown in three different ways: as flow lines; as a top to bottom precedence (e.g. the fact that the Initialize roles of variable plans are executed before the loop because they appear on top of their respective plans, and the Loop Test occurring before the Update and Increment in the loop's body); and a left to right ordering (as evident in the loop body mechanism).

Another problem arises with the slide mechanisms used in some of the plans. What does the slide mean with regard to the Running Total and Counter plans? It may be somewhat clear as to why the slide in the Loop plan is necessary, but the only reason it is present in the other plans seems to be so that the Loop Test can be properly positioned. In addition, what happens if the Loop Test and the Update and/or Increment are positioned at the same level?

\subsection{Generation 5}

Part of the problems encountered by the version in generation 4 seemed to stem from the lack of an analogy for the figures used. There was no intuitive component that allowed users to recognize the meaning of the objects or their characteristics. In addition, as noted above, there was inconsistency in the flow of control.

Figure 16 shows a redesign of the graphical objects used to represent plans. The design was influenced by the Transformer type of toys, with pieces being keyed to fit particular places in other pieces. The flow of control was implied to be strictly left to right and top to bottom.

The design shown in Figure 16 turned out to be too "busy." Figure 17 shows a refinement of the graphics in order to make the objects a bit simpler. Figure 18 shows how some of these objects would fit together. 
There were still some mechanical problems with this design, making the attachment of some of the objects difficult. In addition, it turned out that the left-right, top-bottom control flow was insufficient.

\subsection{Generation 6}

The current version of the visual language is shown in Figure 19. This design was influenced by jig-saw puzzle pieces. The mechanics of this version work out better than the previous version. In addition, control flow is more explicit, following the keyed tabs on each object top-down. Data objects are rectangular shapes with tabs on their left and right sides. Originally, these data objects were placed over a recepticle that contained a "velco" substance for sticking them to. This was later changed to being simply holes that were filled with the data objects.

Figure 20 shows a solution to an averaging problem. During the running of the visual solution, data flow is explicitly provided by having the values actually move from one location to another. For instance, in this example, when a value is entered as an input, the value would automatically be carried by a moving box to the New Value location in the New Value Controlled Loop plan, and on down to the Update location in the Running Total plan. Likewise, the Value from the Running Total plan and from the Counter plan would be carried to the appropriate locations in the Compute plan. The Value in the Compute plan would be carried down to the Output plan.

There are still problems to be overcome in this current version of the visual language. For instance, the issue of nesting plans has still not been fully addressed. In addition, there is still some concern that the shapes of the plans are not adequate in their ability to evoke understanding by the user.

Despite the difficulties with the current version, what is clear at this point is that the use of a visual language is effective.

\section{Future Steps}

There are several issues still to be resolved with BridgeTalk. These issues fall into the general areas of visual appearence, implementation, and usage. We take up each topic separately.

The most problematic issue with the visual appearence of BridgeTalk is the difficulty of expressing nesting. In particular, the current version can visually express only one level of nesting, and that one level with only a fixed number of contained elements. The problem is that there must be only a small finite amount of space to put a contained element if the parent icon is visually containing them. Inevitably, we will need to go to some sort of grow/shrink scheme for the contained elements. We have 
resisted this because it is inherently disturbing and confusing unless the grow/shrink transistion is smooth and obviously reversable. Most visual languages address this problem by using small icon elements that expand to windows. This solution is unacceptable due to our experience suggesting the importance of icon shapes suggesting the icon usage.

The BridgeTalk implementation is still incomplete. In particular, we still have not completed the browsers that allow a user to navigate the structures showing plan inheritance and implementation, as pictured in Figures 8 and 9. We will have a challenge in distinguishing this representation from the specific plan icons used at the surface level. On the otherhand, we feel these structures are a crucial tool for teaching students to understand the management of abstraction.

The final area of future work suggested by this work is the most complex: how is this tool and approach best used? In our attempt to tease apart the expression of intentions to a computer, we have become increasingly suspicious of the glib discussion of "top-down design" found in most programming textbooks. In particular, the design of BridgeTalk shows that programming design involves many different mappings, including: informal to formal, declarative to procedural, goals to plans and processes, natural language to Pascal, linear structure to tree structure, and weakly constrained to strongly constrained. We believe that programming texts do their students a disservice by presenting a design model that at best ignores the differences between novices and experts, and at worst is completly unrelated to actual programming practice.

\section{Conclusions}

We have reported on BridgeTalk, a new approach to visual languages for novice programmers. BridgeTalk is based on actual data about how novices learn to program. It allows novices to program with programming plans, and focuses novices on the interactions between plans, not on the implementation details for a particular plan. Beginning with plans as a basis for a novice programming language, we were forced to develop a programming formalism that could deal with multiple levels of detail, merged plan implementations, and interrelationships between plans. Finally, we used six "design, implement, test, and redesign" cycles to develop a specific visual representation for the language.

A key contribution of this work is the systematic support for a plan-like view of programming. We feel that such support is essential if programming languages are to allow a programmer, novice or expert, to work with programming constructs that reflect actual world semantics. Unfortunately, "high level programming language" has 
come to mean more abstract data structures and more formal semantics. We advocate, and have demonstrated the feasability of, "high level languages" that reflect the semantics of real-world objects.

Consistant with a language that makes a richer connection to the semantics of real-world objects, our language provides novices with a pathway from experience in the real-world, to real-world plans, to standard programming language constructs. Current software systems force a user to either use the system as is, or become a programmer. BridgeTalk illustrates an approach that provides a smoother transition with many intermediate steps along the way. That is, programming strictly with plans simplifies programming by allowing users to draw on their knowledge of real-world situations, but limits users to those plans provided in the system. Because the internals of the plans can be examined, modified, and specialized, users can extend the power of their system beyond the predefined plans.

One of the most important lessons of this work is the criticality of empiricial work in the design of visual languages. Without the extensive empirical work documented in this report, our language would be much poorer. Furthermore, the language would more than likely be unsuitable for use with novices. There is no reason to believe that languages designed based on purely formal and intuitive arguments will be usable. Until a suitable theory of visual interface design emerges, we feel that computer scientists must submit their visual languages to the test of real users under realistic circumstances. Not only will this produce happier languages, we believe, but also better science.

\section{References}

Bonar, J., Cunningham, R., Beatty, P., \& Riggs, P. [1987]. Bridge: Intelligent Tutoring With Intermediate Representations. Technical Report, Learning Research and Development Center, University of Pittsburgh, Pittsburgh, PA 15260

Bonar, J., and Soloway, E. [1985]. Pre-programming Knowledge: A Major Source of Misconceptions in Novice Programmers. Human-Computer Interaction, 1.

Bonar, J., Weil, W., \& Jones, R. [1986]. The Programming Plans Workbook. Technical Report, Learning Research and Development Center, University of Pittsburgh, Pittsburgh, $:$ A 15260

Cunningham, R., Corbett, J., \& Bonar, J. [1987] The Chips Technical Report. Technical Report, Learning Research and Development Center, University of Pittsburgh, Pittsburgh, PA 15260 
Liffick, B. [1987]. A Visual Programming Environment for Novices. Technical Report, Learning Research and Development Center, University of Pittsburgh, Pittsburgh, PA 15260

Rich, C. [1981]. A Formal Representation for Plans in the Programmer's Apprentice. Proceedings of the 7th International Joint Conference on Artificial Intelligence, Vancouver, Canada, 1981. Also in Artificial Intelligence and Software Engineering, Rich, C. and Waters, R.C (ed.). Morgan Kaufmann Publishing, 1986

Shrobe, H. [1979]. Dependency Directed Reasoning for Complex Program Understanding. PhD Thesis. MIT/AITR-503, April 1979

Soloway, E. Bonar, J., \& Ehrlich, K. [1983]. Cognitive Strategies and Looping Constructs: An Empirical Study. Communications of the ACM, 26, November.

Soloway, E., \& Ehrlich, K. [1984]. Emperical Studies of Programming Knowledge. IEEE Transactions of Software Engineering, SE-10

Spohrer, J., Soloway, E., \& Pope E. [1985]. A Goal/Plan Analysis of Buggy Pascal Programs. Human-Computer Interaction, 1

Waters, R.C. [1978]. Automatic Analysis of the Logical Structure of Programs. PhD Thesis. MIT/AI/TR-492, December 1978

Waters, R.C. [1985]. The Programmer's Apprentice: A Session with KBEmacs. IEEE Transactions of Software Engineering, SE-11

Winograd, T. [1979]. Beyond Programming Languages. Communications of the ACM, July 1979. Also in Interactive Programming Environments, Barstow, D. et al (ed.), McGraw-Hill, 1984 


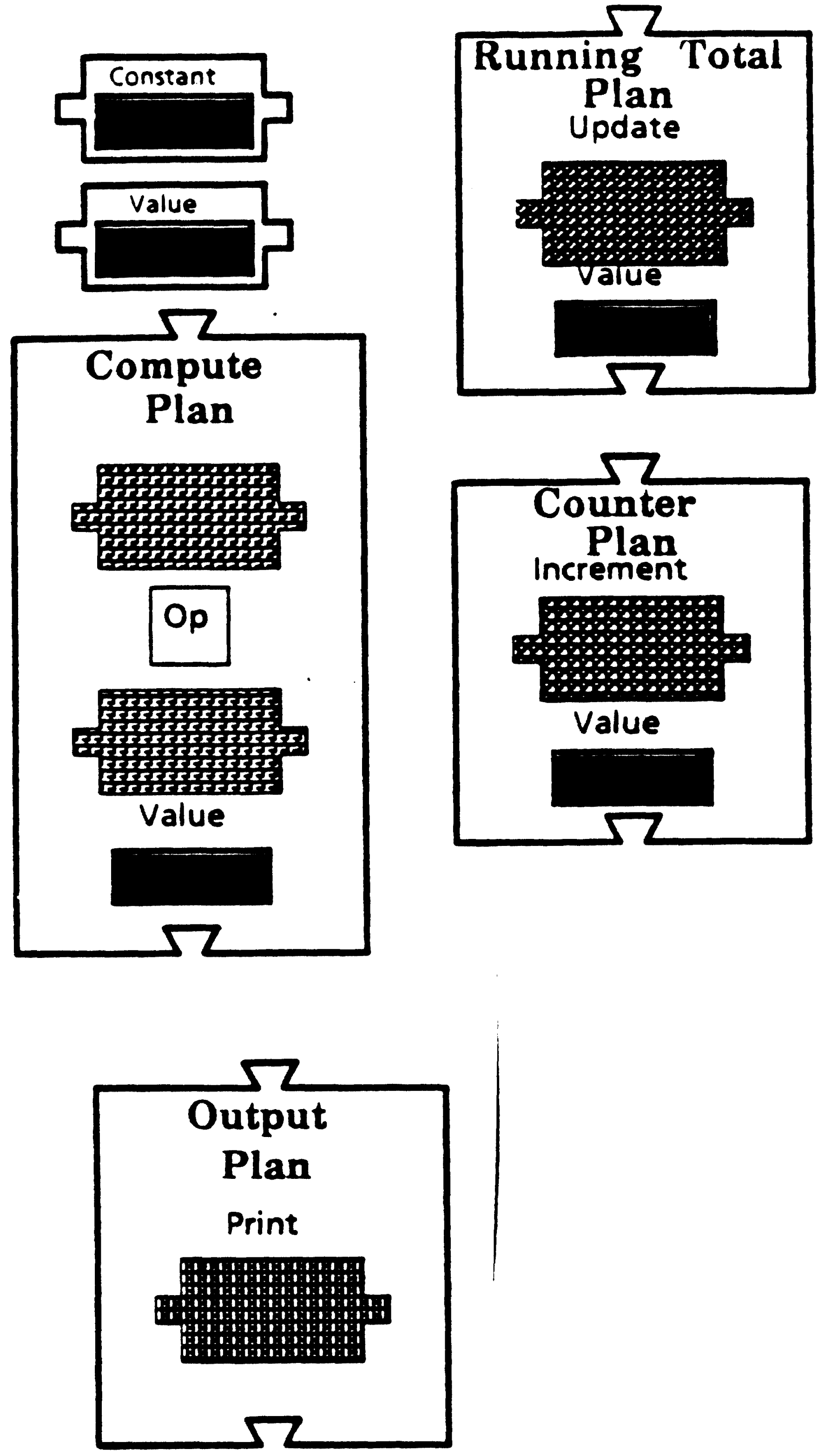

FIOUPE 1. 


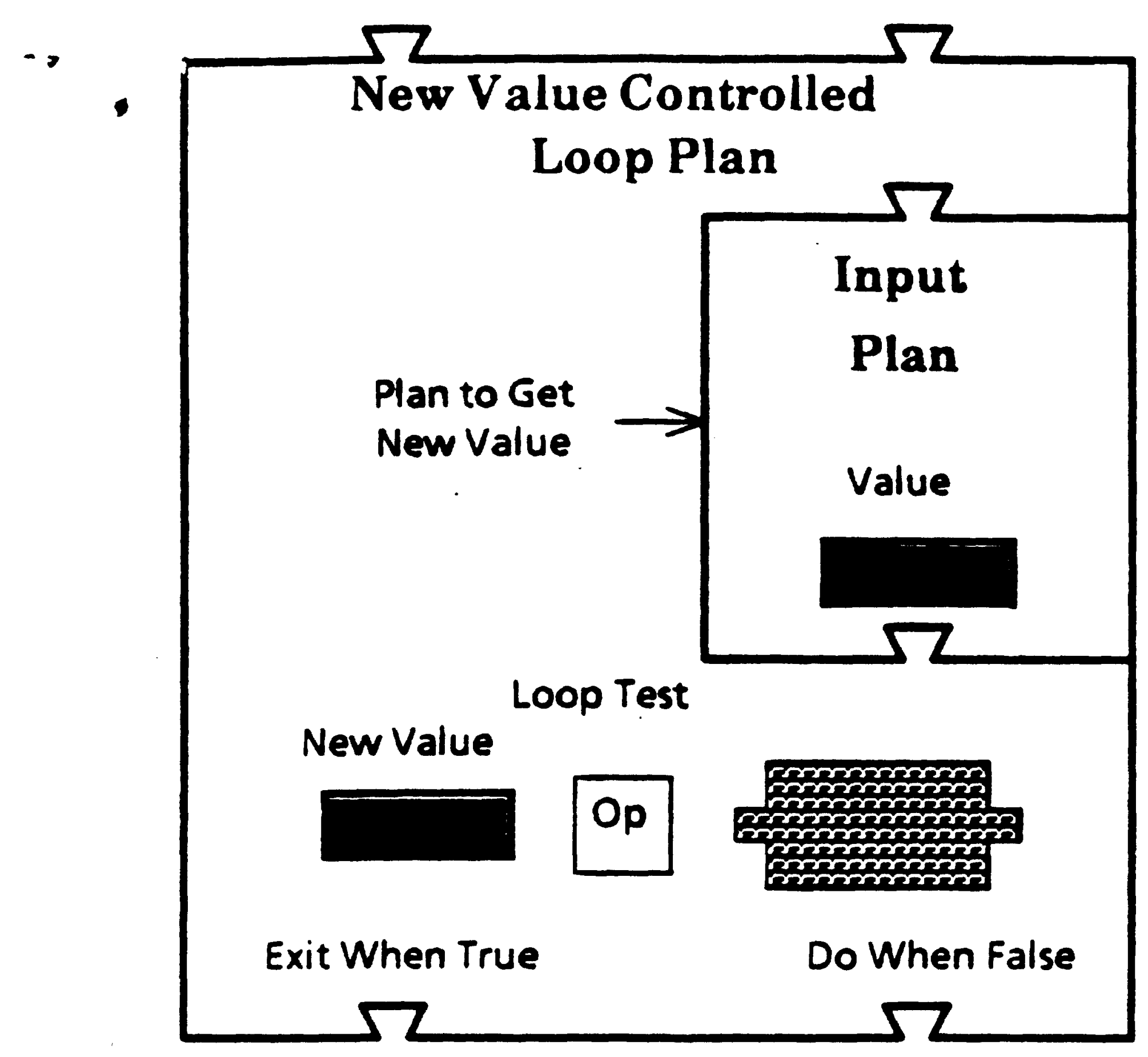

PICISE 2 


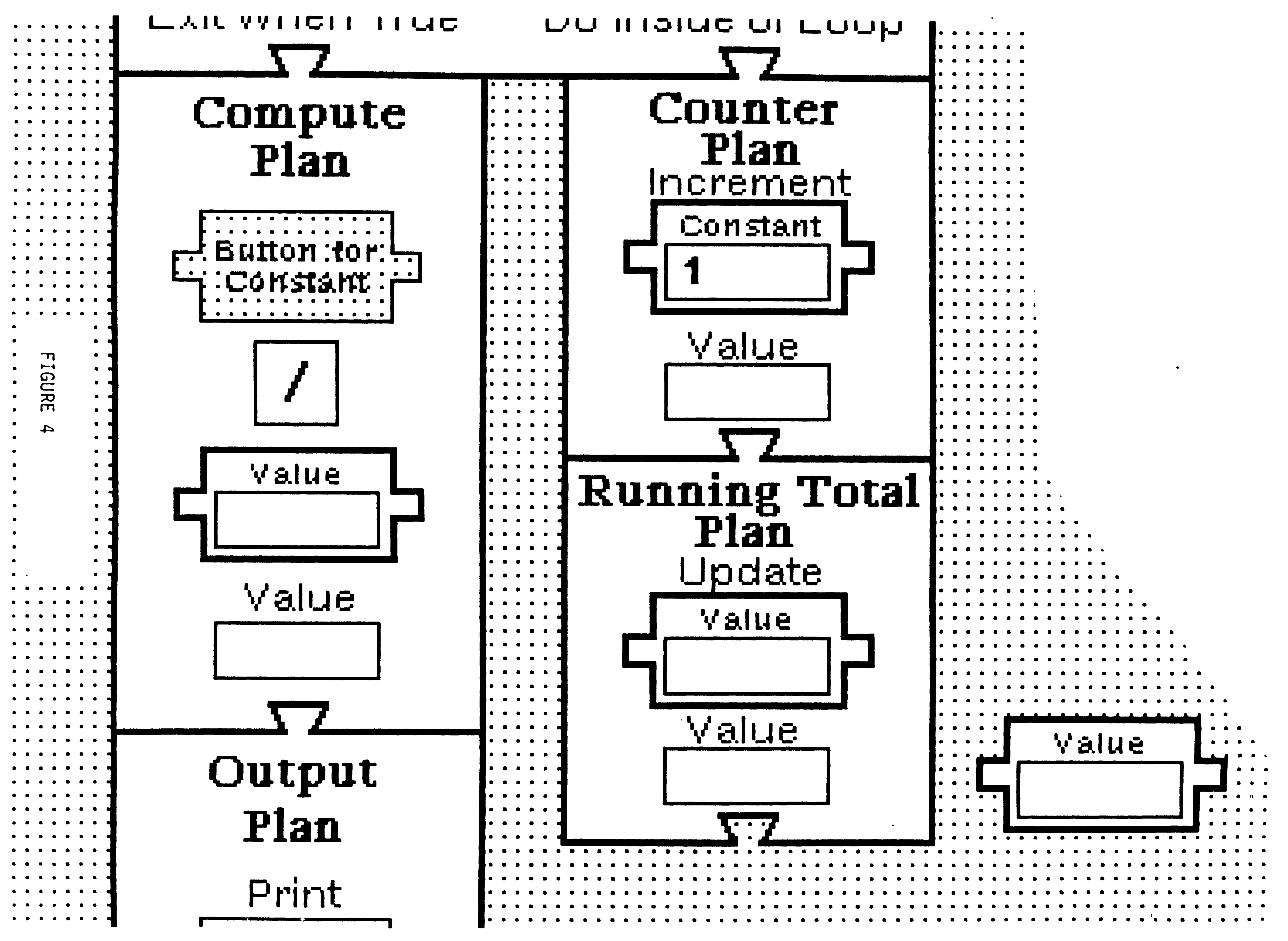




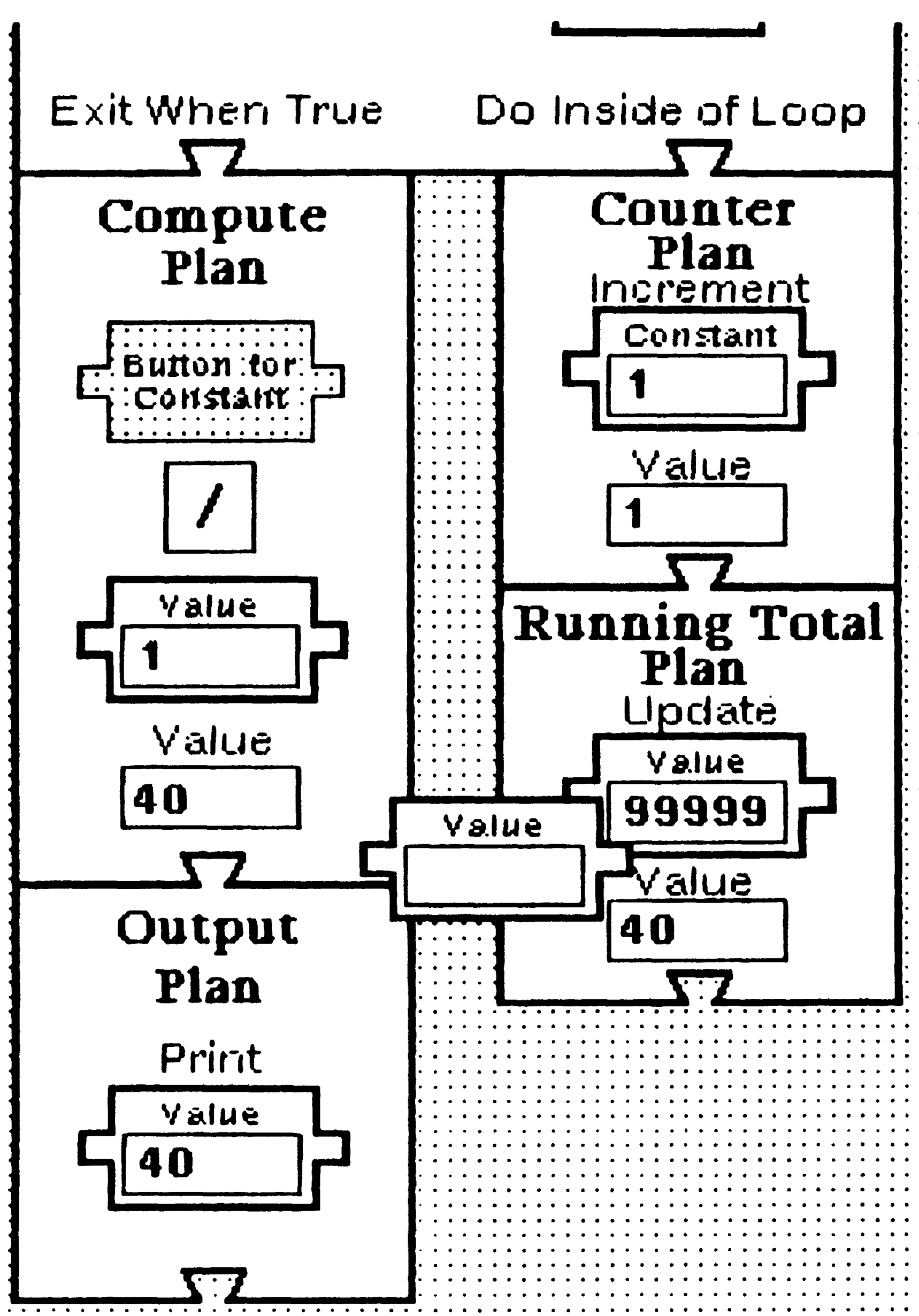




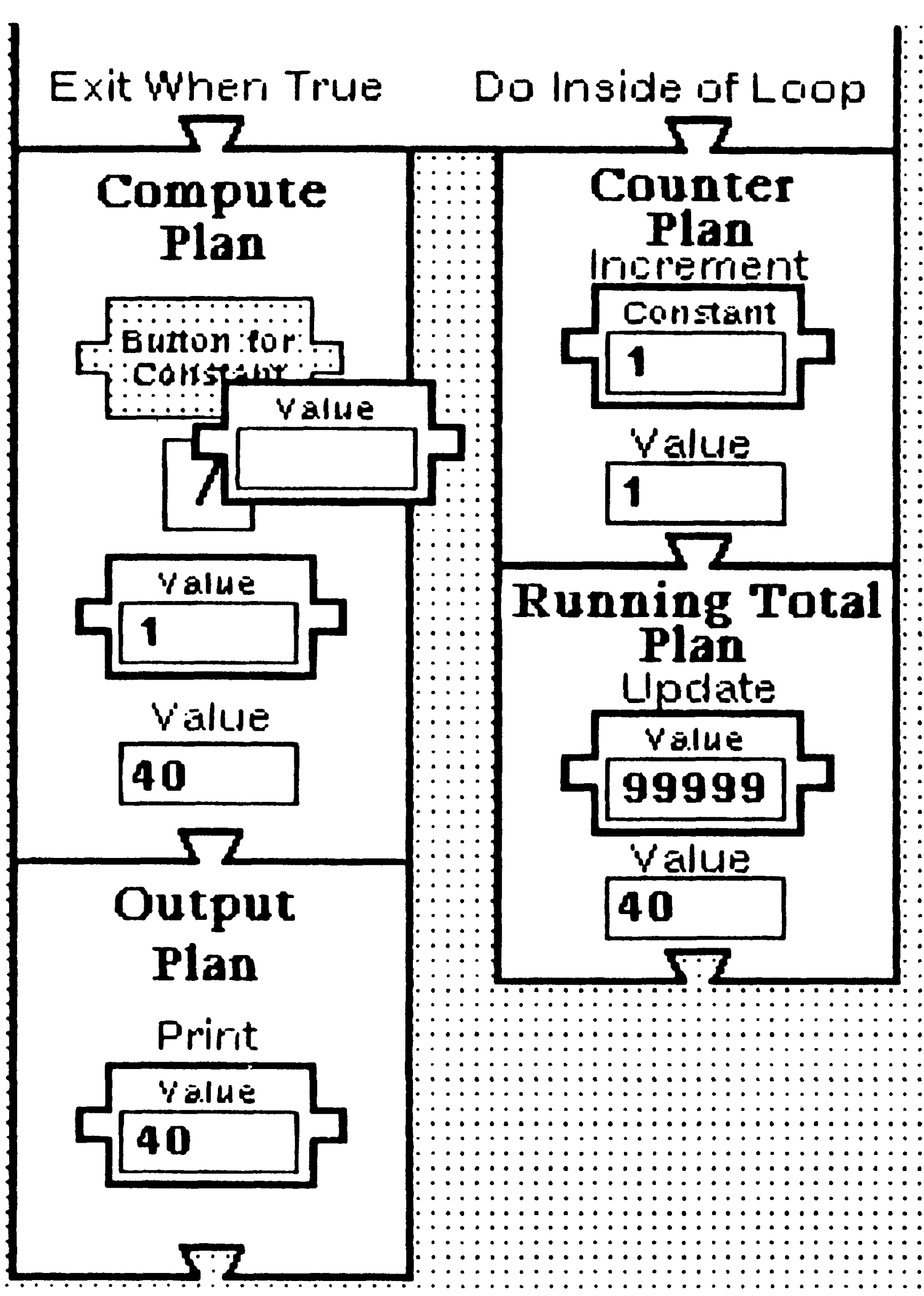


LOOP ACTION PLAN

RUNNING TOTAL PLAN

PARENTCLASS:

LOOP ACTION

SLOTS:

value.TOTAL..$<>$

ADDEND -. $<>$

INITIAL -. O*

INITIALIZATION:

TOTAL <--- INITIAL

EXECUTION:

TOTAL <--- TOTAL + ADDEND

\section{CONSTANT RUNNING TOTAL PLAN}

PARENTCLASS:

RUNNING TOTAL

SLOTS:

ADDEND.INCREMENT -- (CONSTANT)

\section{COUNTER PLAN}

CLASS:

CONSTANT RUNNING TOTAL

SLOTS:

TOTAL.COUNT.$-<>$

ADDEND.INCREMENT -- 1 * 


\section{ENDING VALUE AVERAGING LOOP PLAN}

SLOTS:

TOTAL -- (RUNNING TOTAL)

COUNT -- (COUNTER)

LOOP -- (SENTINEL LOOP: BODY <-- [TOTAL,COUNT])

value.AVERAGE -- (AVERAGE: DIVIDEND <--TOTAL; DIVISOR <-- COUNT)

OUTPUT -- (OUTPUT: OUT <-- AVERAGE; MESSAGE <-- "The average is ")

EXECUTION

LOOP $<==$ Execute

AVERAGE $<==$ Execute

OUTPUT $\angle==$ Execute

\section{SENTINEL LOOP PLAN}

PARENTCLASS:

NEW VALUE CONTROLLED LOOP

SLOTS:

NEWVALUE -- (INPUT: MESSAGE <-- "Enter a new value")

TESTVALUE.SENTINEL -- (CONSTANT)

WHENTOHALT -. ' = '*

\section{NEW VALUE CONTROLLED LOOP PLAN}

SLOTS:

NEWVALUE.$-<>$

TESTVALUE $--<>$

WHENTOHALT -- $<>$

TEST -- (TEST: TESTVALUE1 < - NEWVALUE; TESTVALUE2 <-- TESTVALUE;

RELATIONALOPERATOR <-- WHENTOHALT)

BODY .. $<>$

EXECUTION:

Loop

NEWVALUE $<==$ Execute

TEST $<==$ Execute

If value.TEST is False then Exit

Endloop

BODY $<==$ Execute 


\section{COMPUTE PLAN}

SLOTS:

value.RESULT -. $<>$

OPERAND1 $--<>$

OPERAND2 -- $<>$

OPERATOR $--<>$

EXECUTION:

RESULT <-- OPERATOR(OPERAND1,OPERAND2)

\section{AVERAGE PLAN}

PARENTCLASS:

COMPUTE

SLOTS:

RESULT.AVERAGE $--<>$

OPERAND1.DIVIDEND -- $<>$

OPERAND2.DIVISOR -- $<>$

OPERATOR -- $\%$

\section{TEST PLAN}

PARENTCLASS:

COMPUTE

SLOTS:

OPERAND1.TESTVALUE1.$-<>$

OPERAND2.TESTVALUE $2--<>$

OPERATOR.RELATIONALOPERATOR $--<>$

INPUT PLAN

SLOTS:

value.IN $--<>$

MESSAGE $--<>$

EXECUTION

FIGURE 7 , part 2 of 3 
OUTPUT(MESSAGE) $<==$ Execute

INPUT(IN) $<==$ Execute

\section{OUTPUT PLAN}

SLOTS:

value.OUT -- $<>$

MESSAGE $--<>$

EXECUTION:

OUTPUT(MESSAGE) $<=$ = Execute

OUTPUT(OUT) $<==$ Execute

FIGURE 7 , part 3 of 3 


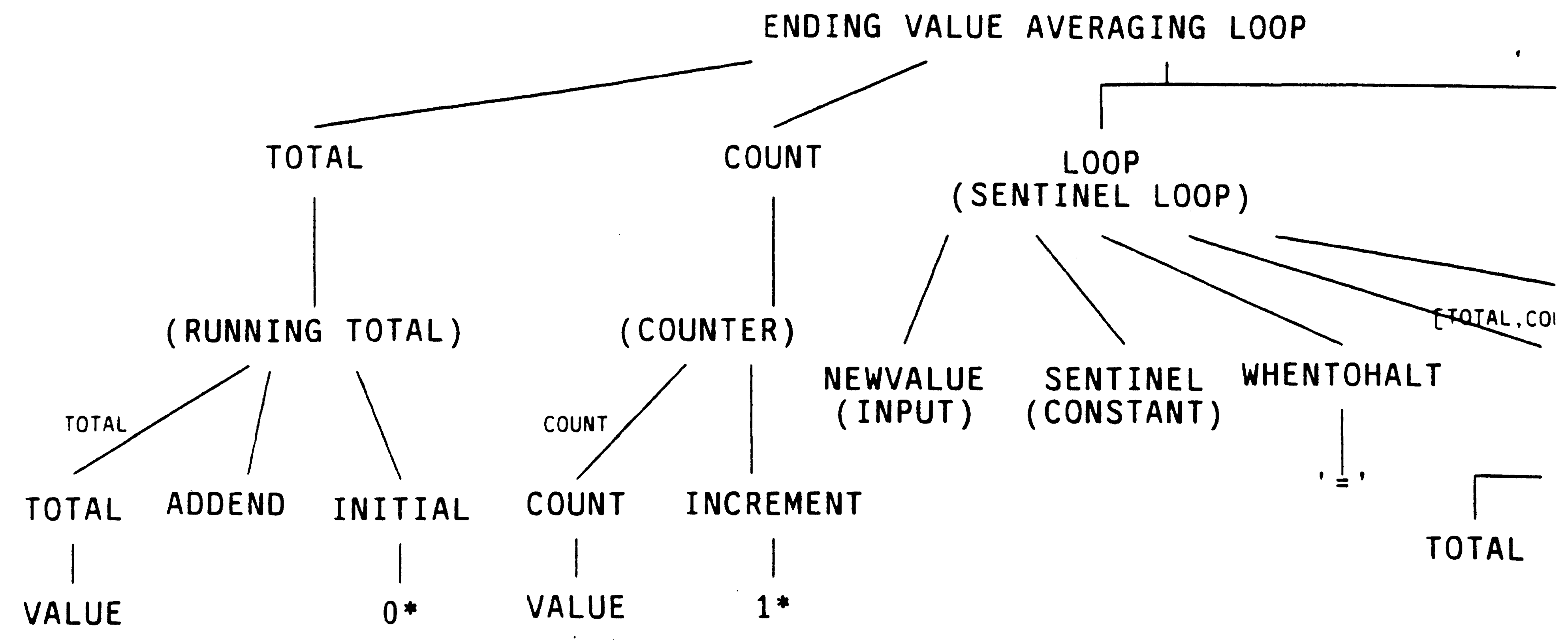

FIGURE 8 , part 1 of 2 


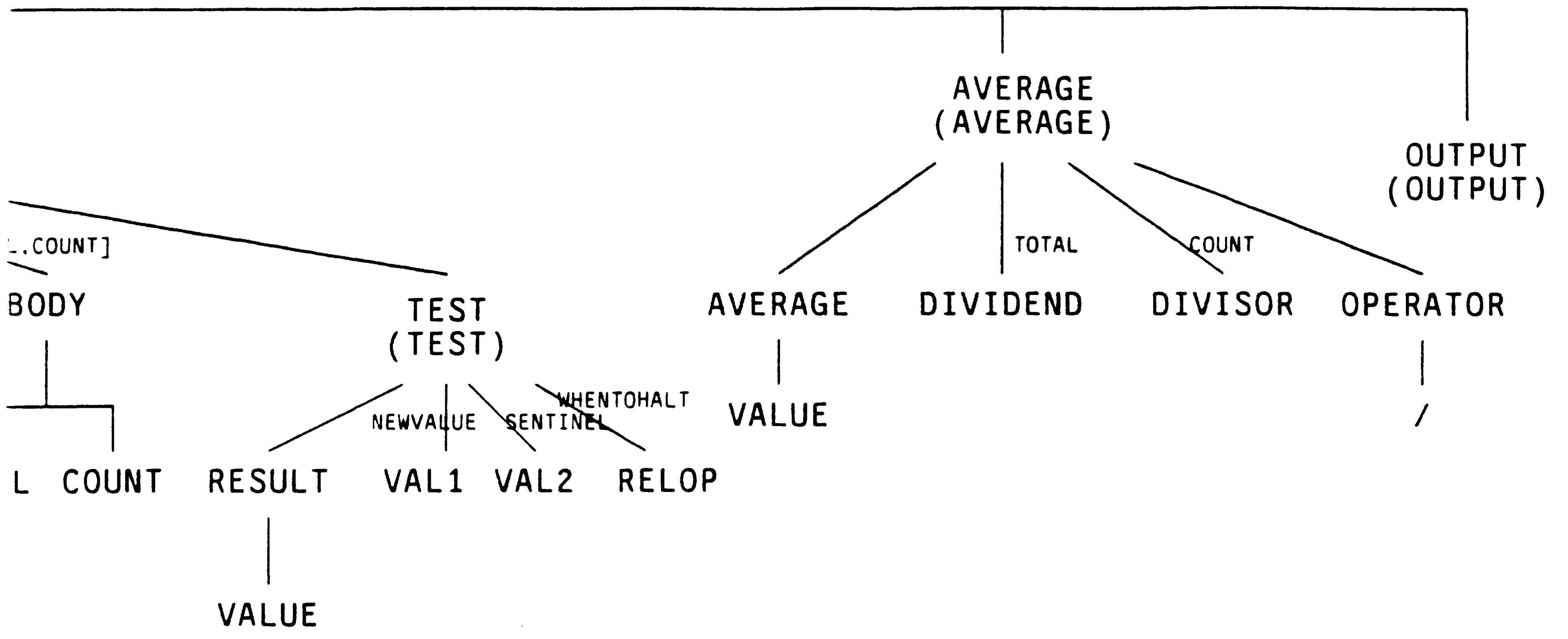

FIGURE 8 , part 2 of 2 


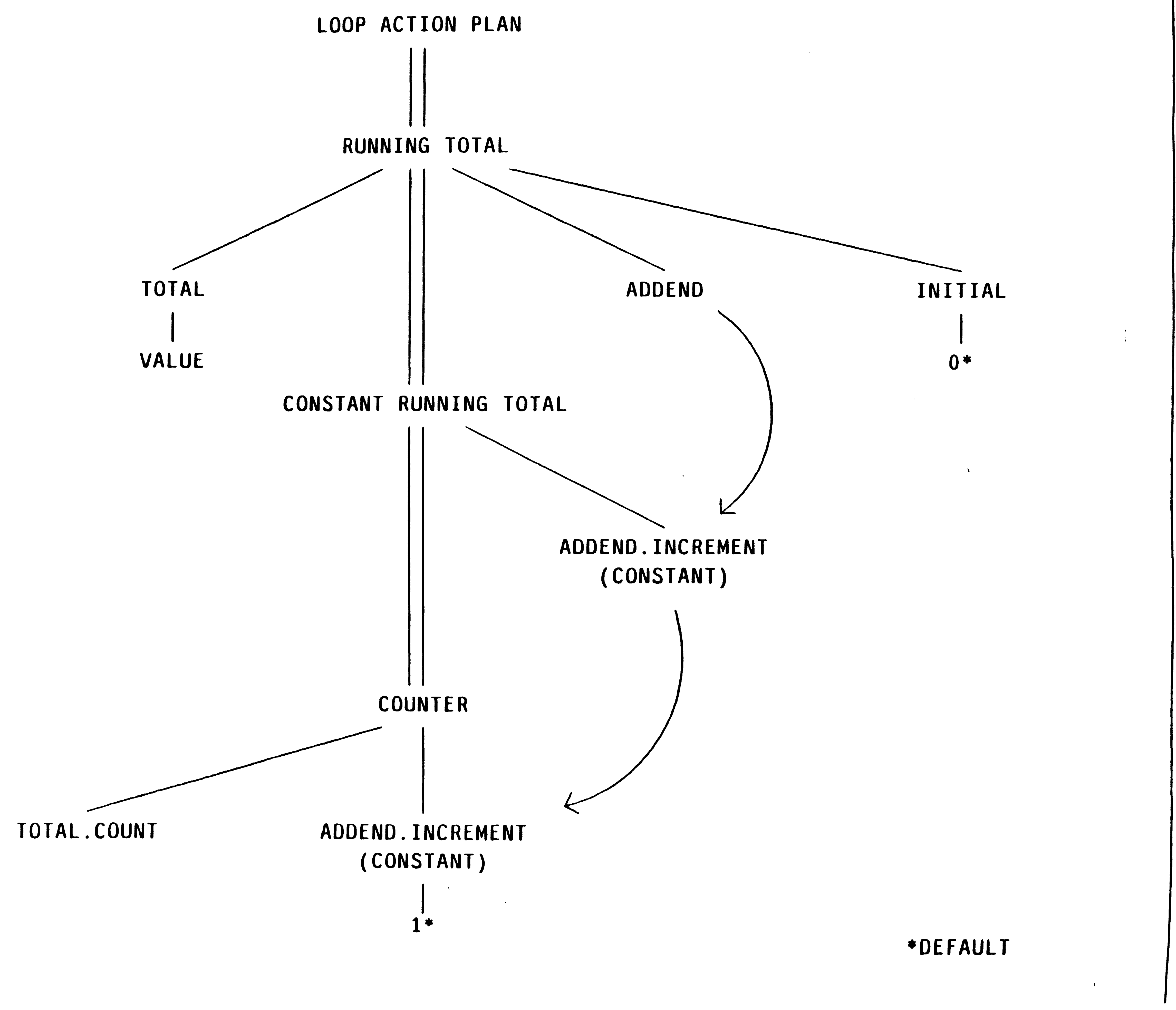




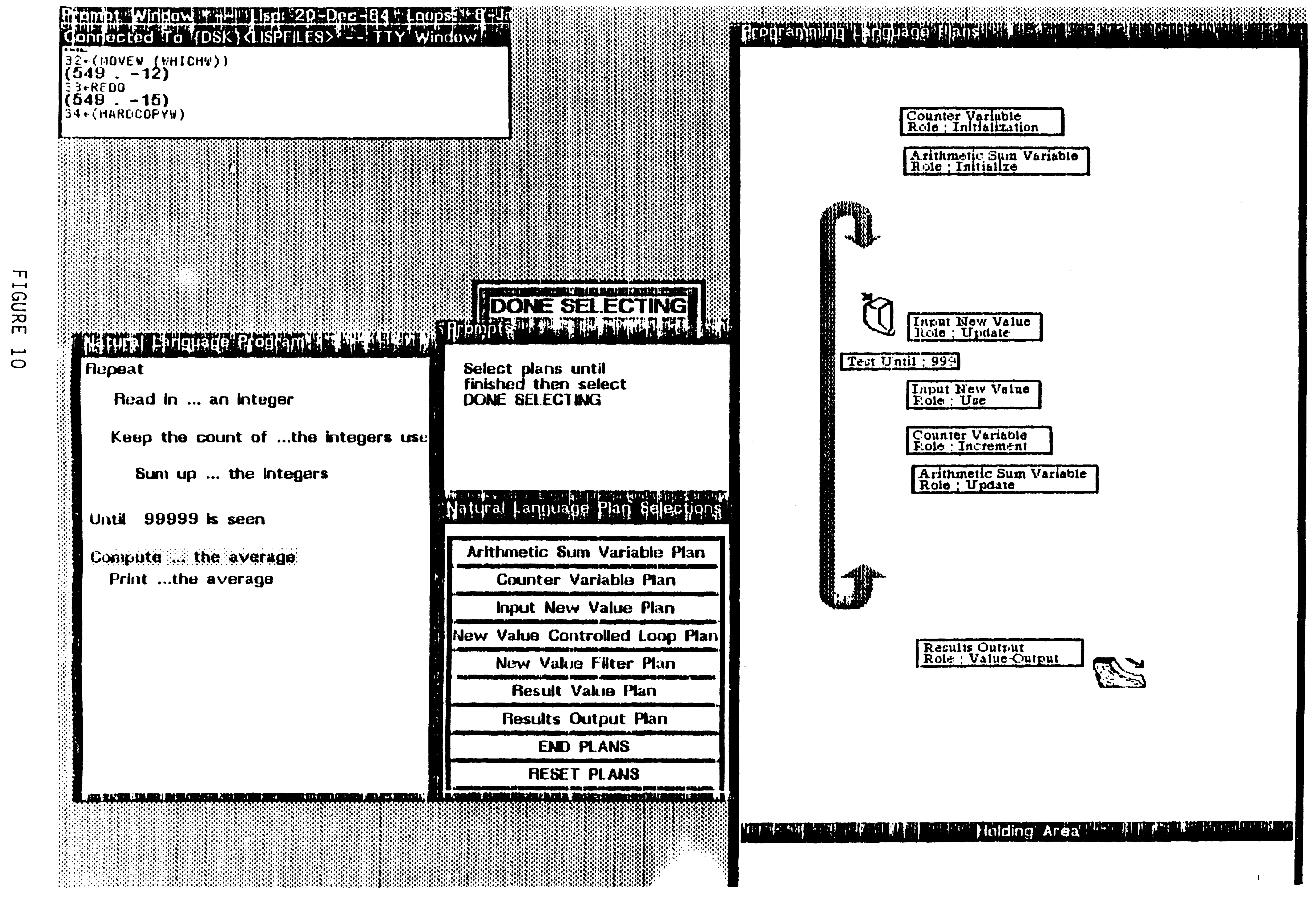




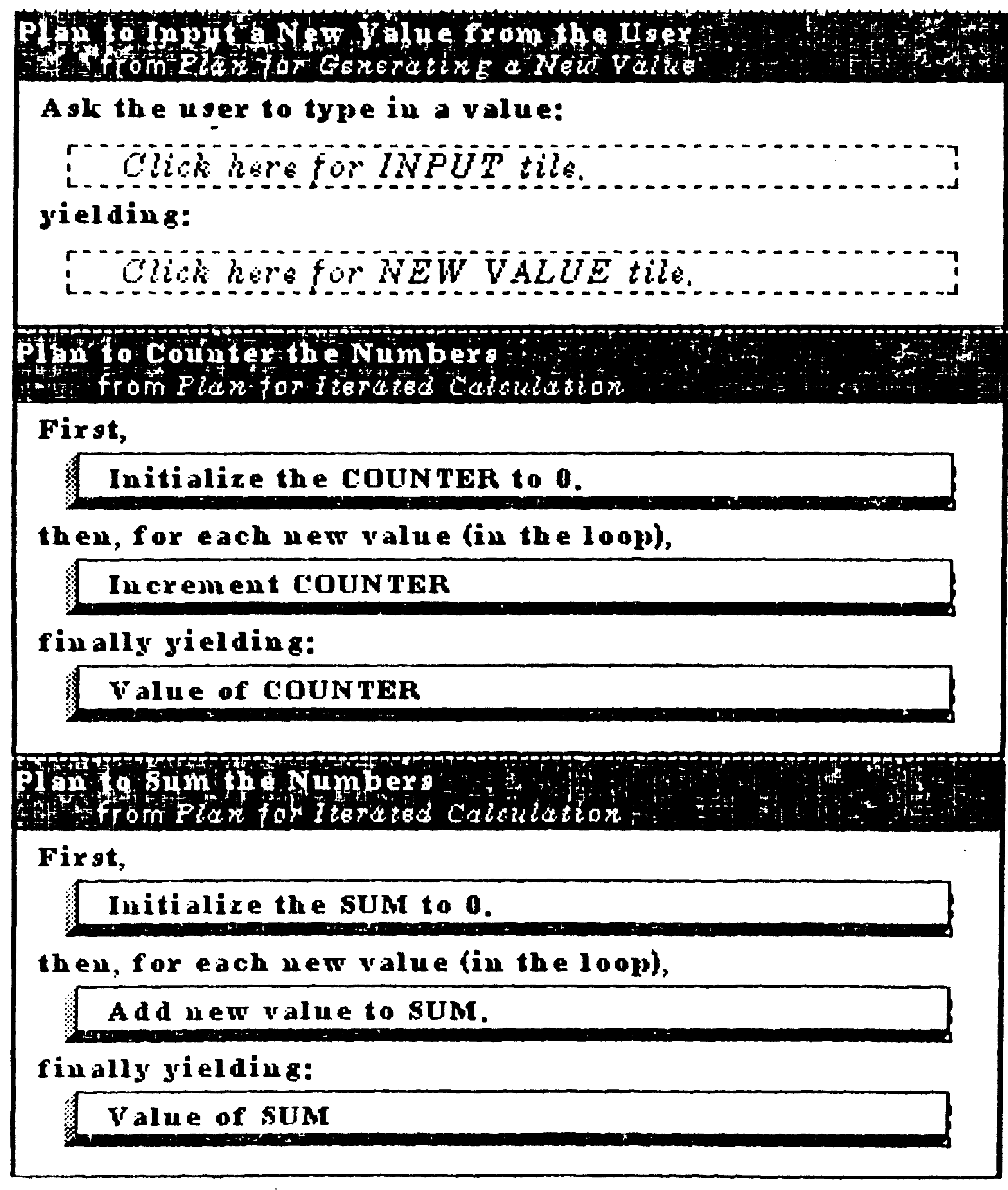




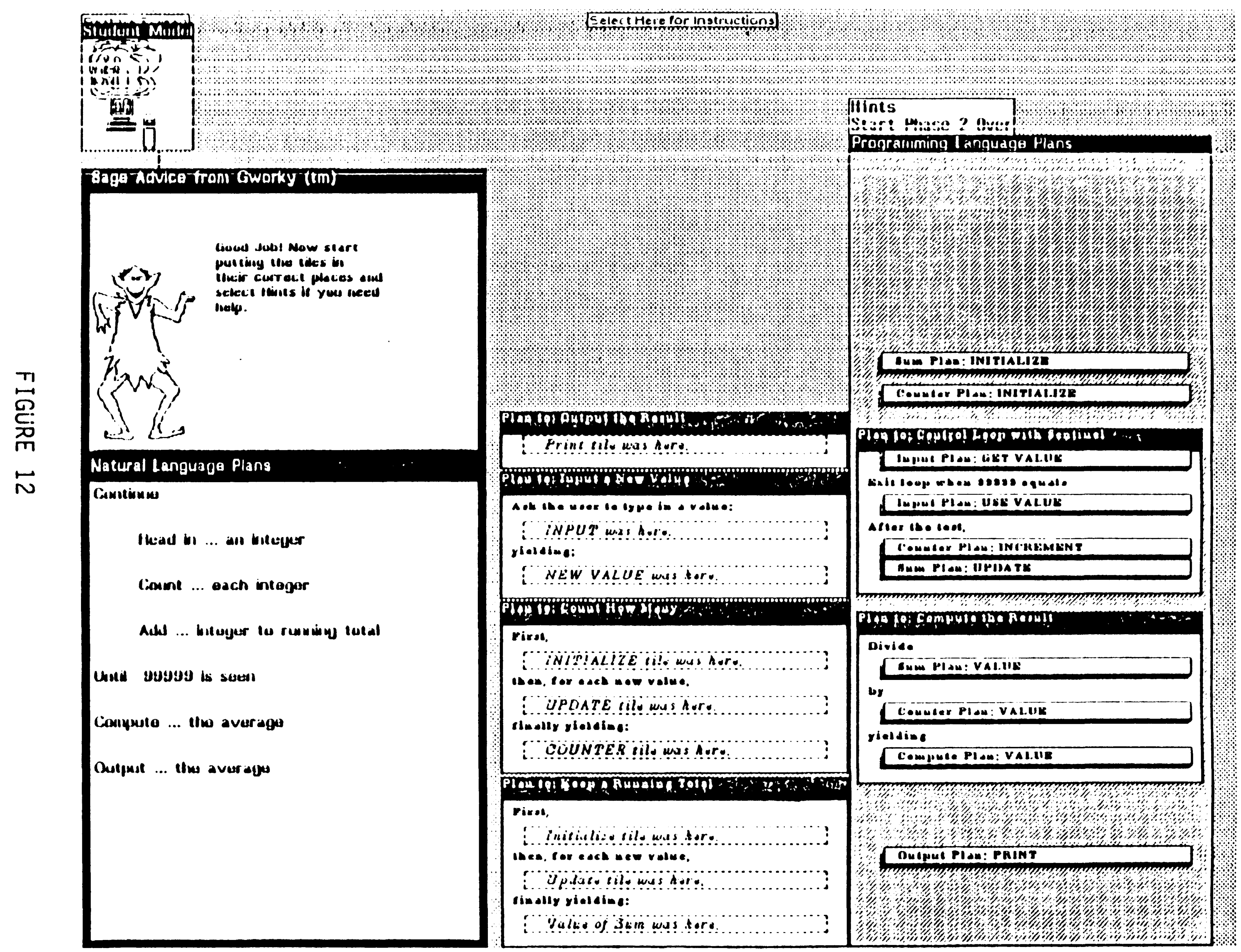


FIGURE 13

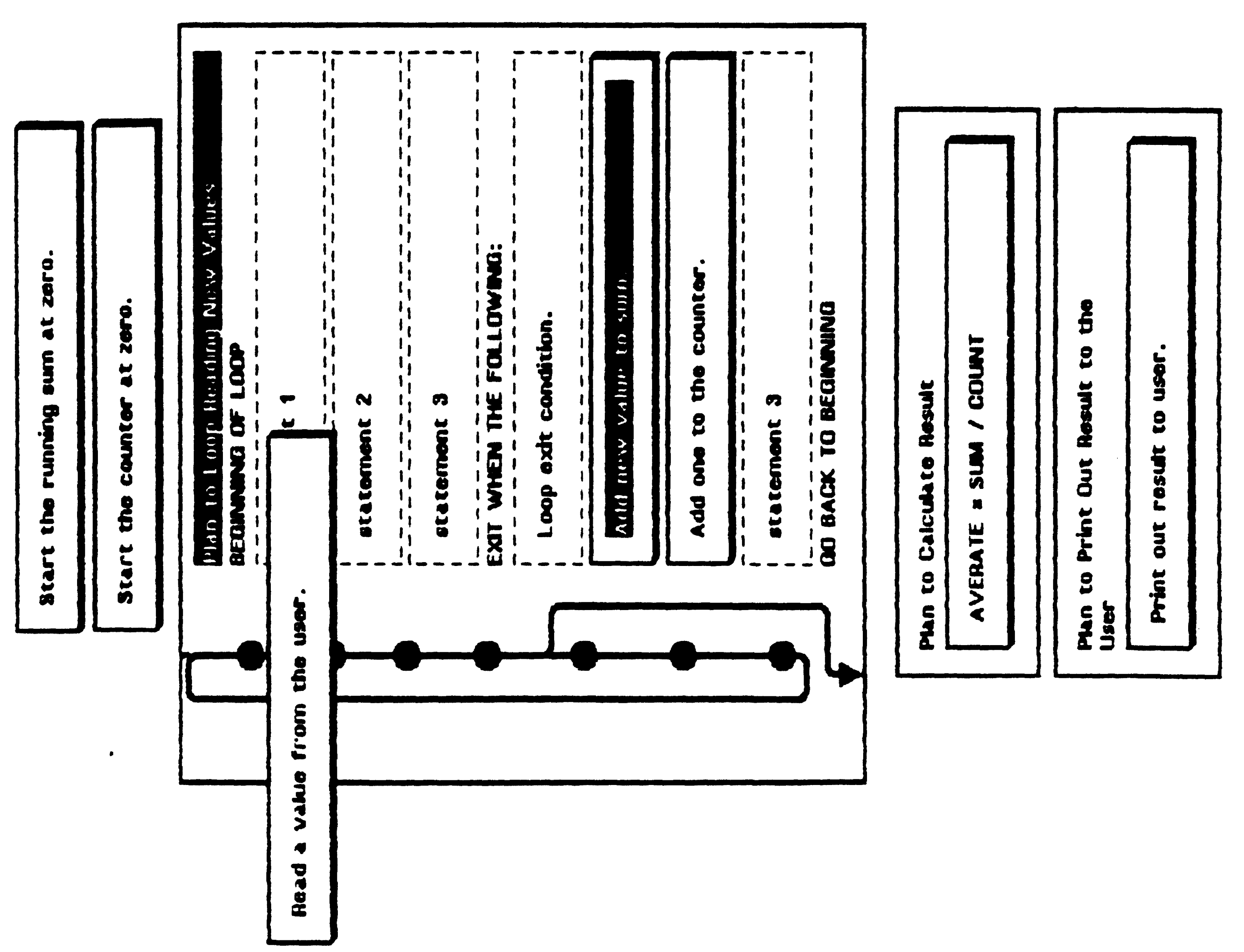




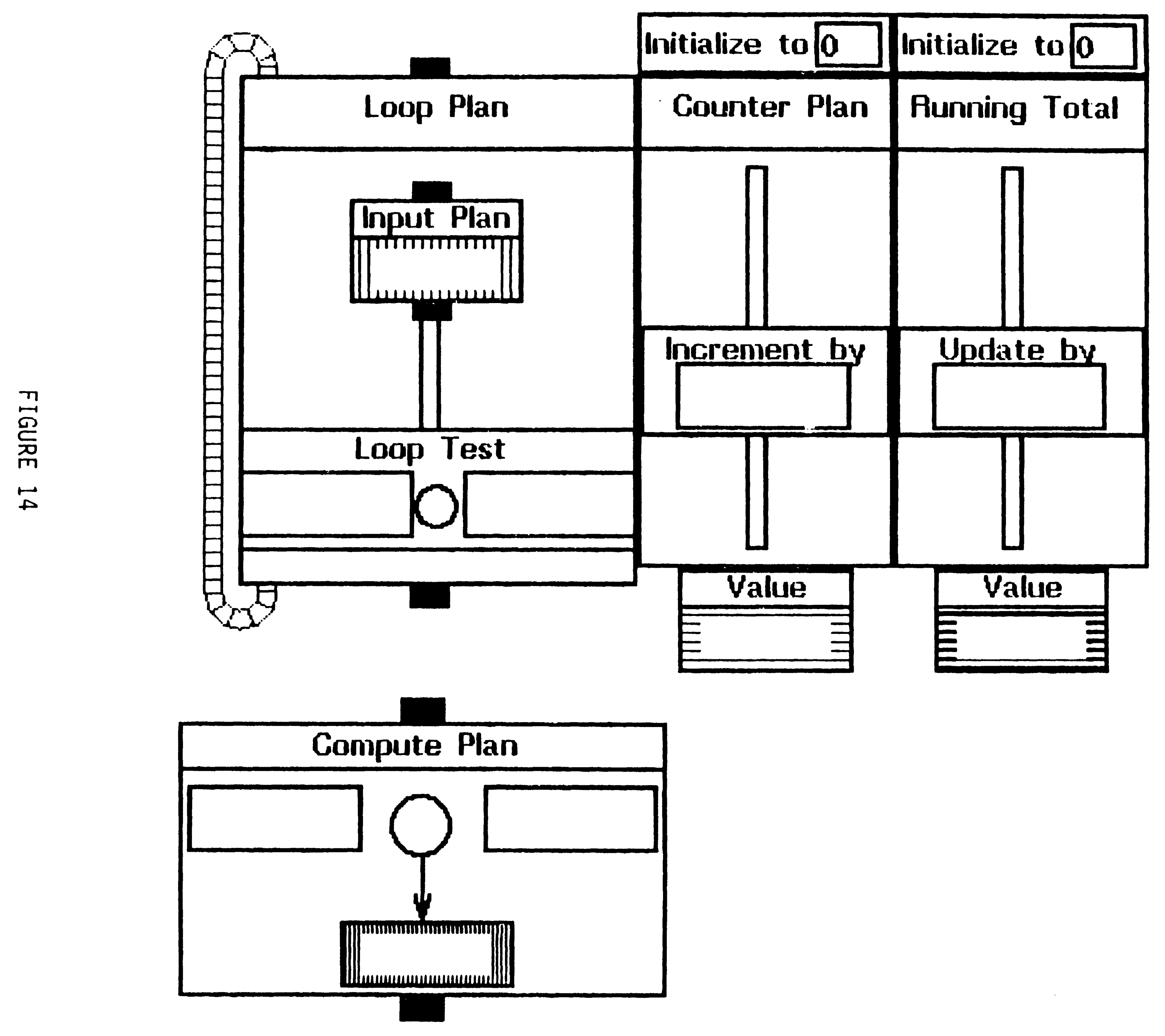




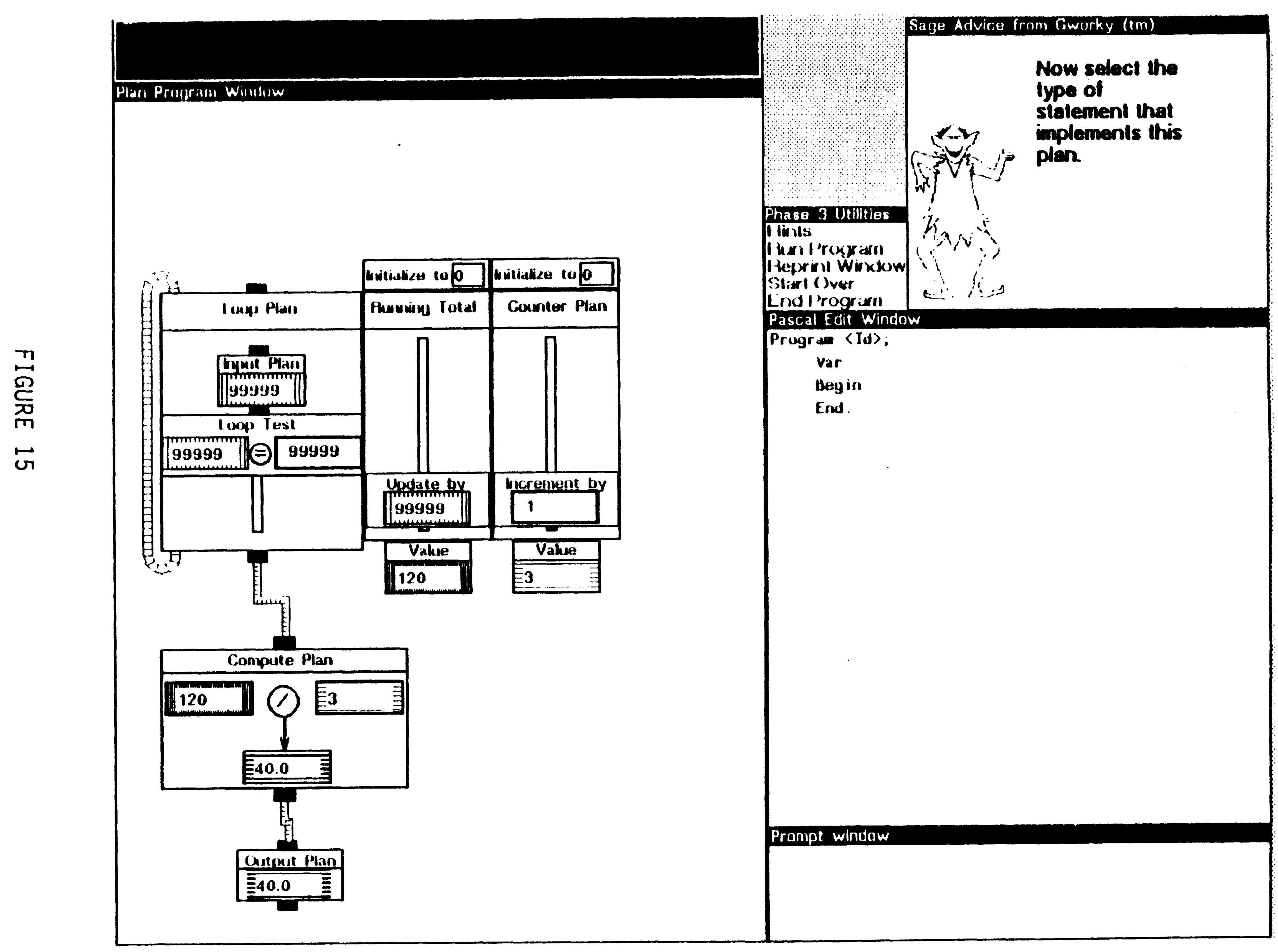



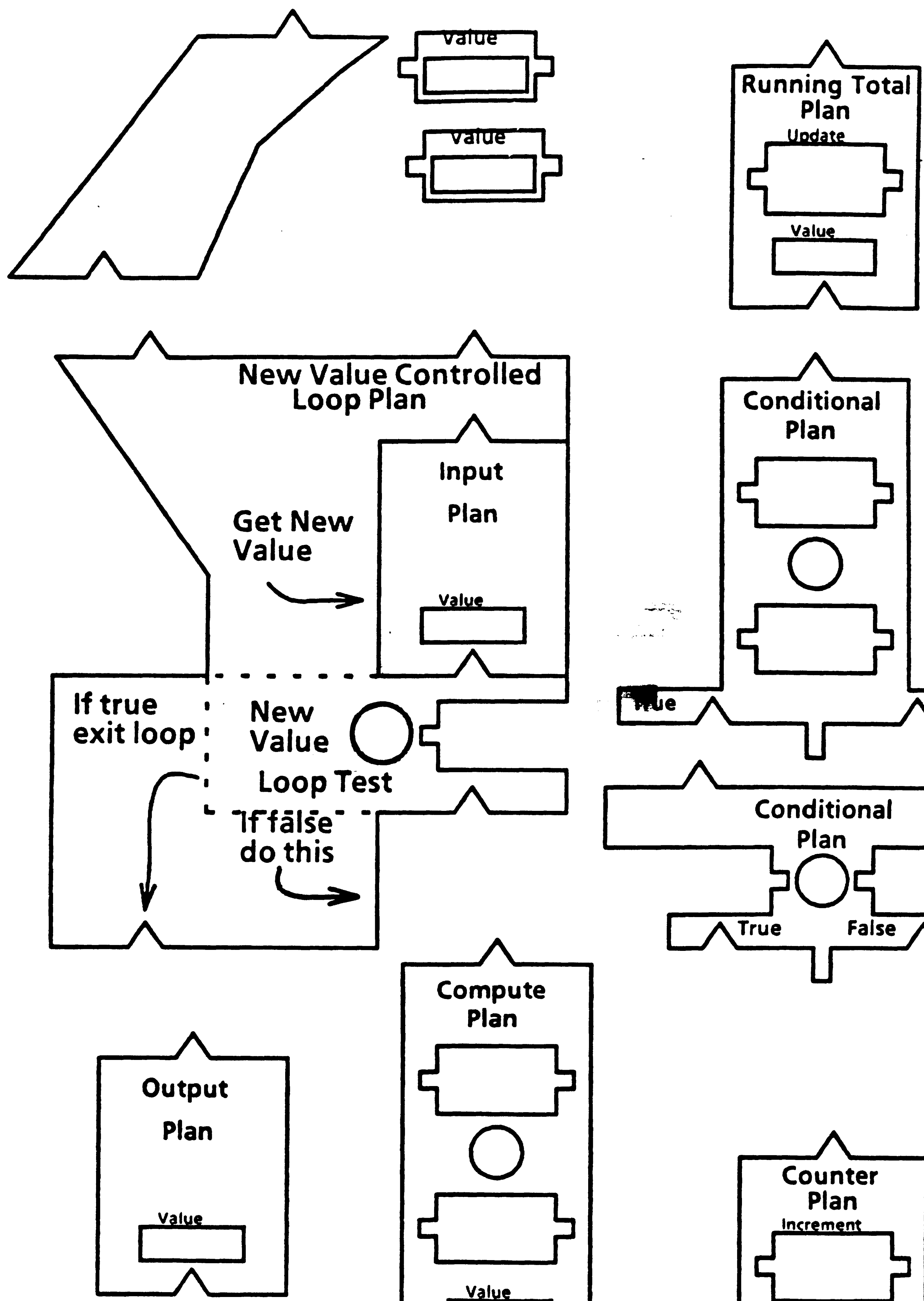

trolled oop Plan

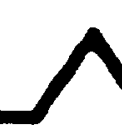

Input

et New

Plan

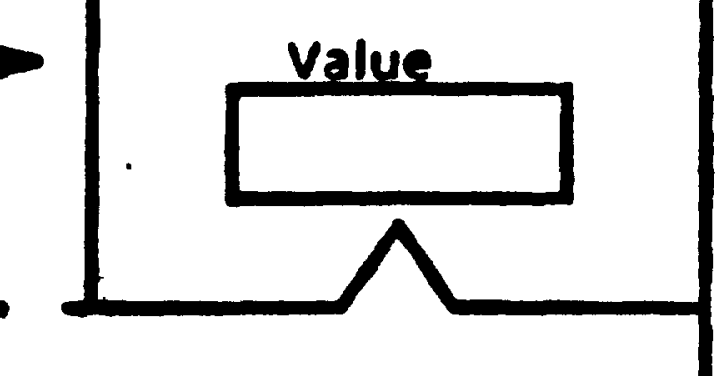

If true, New

exit loop: Value
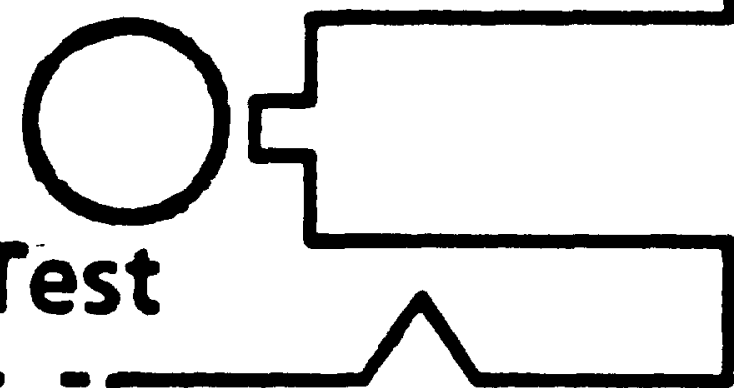

$-$
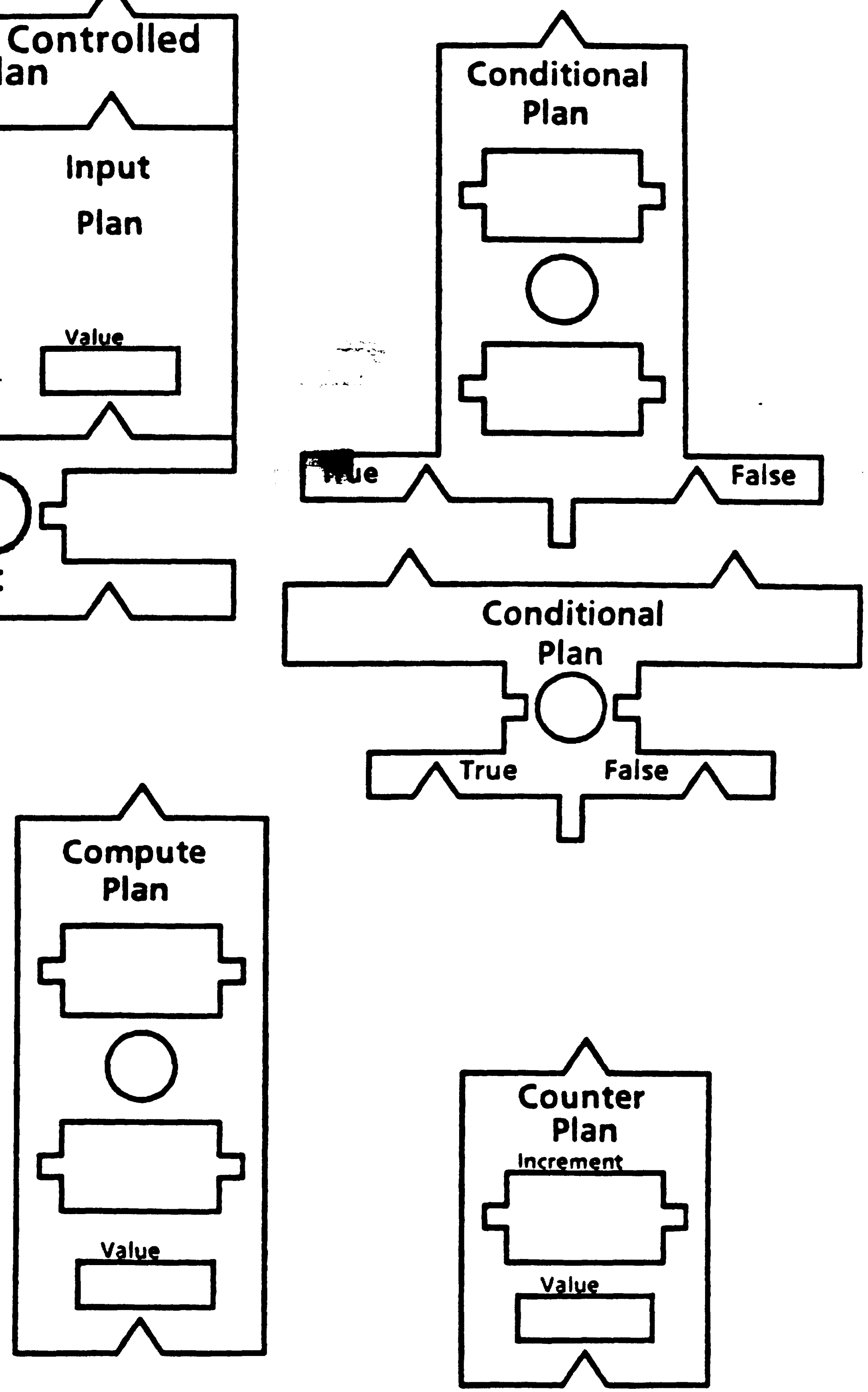

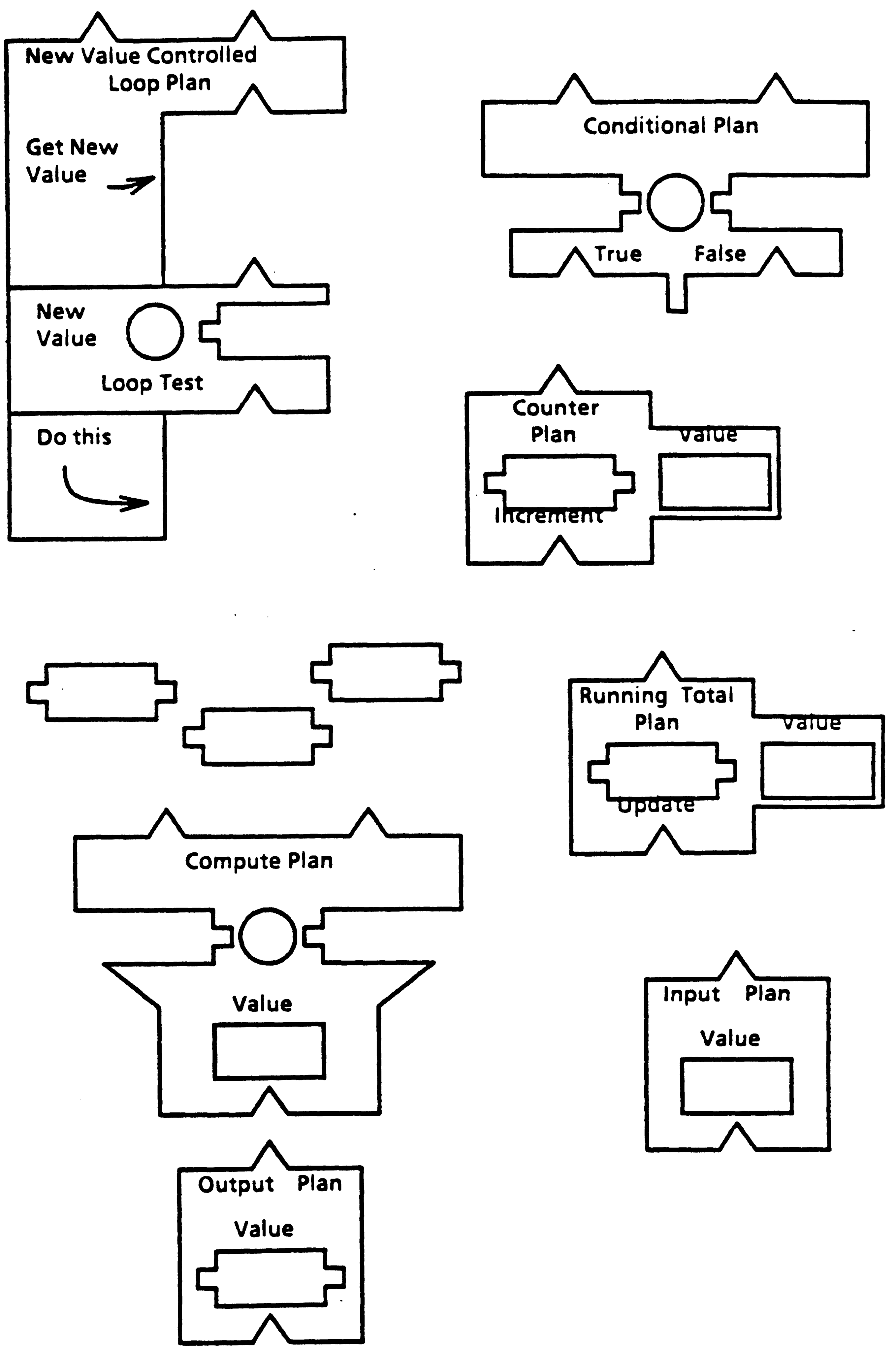


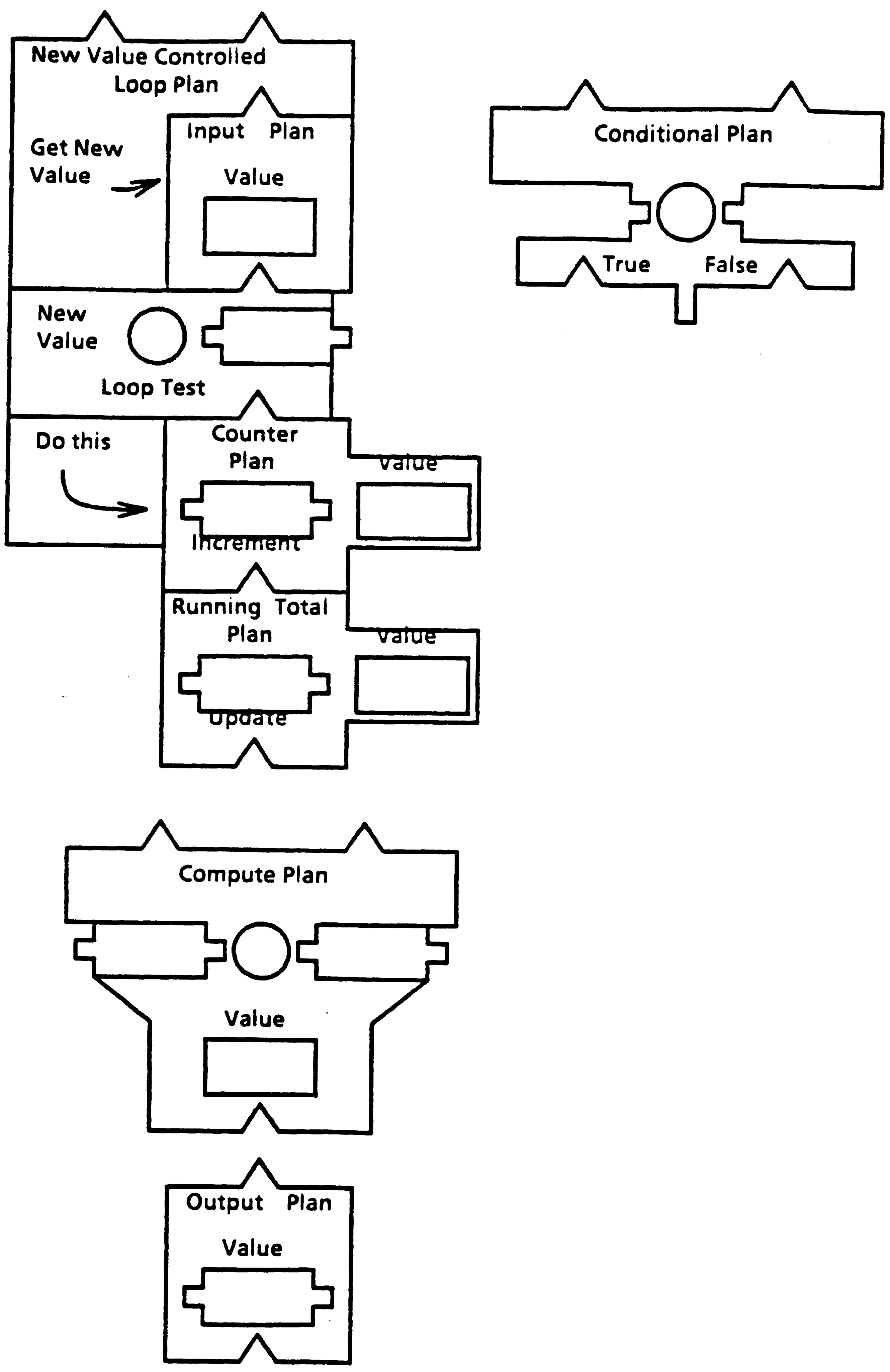




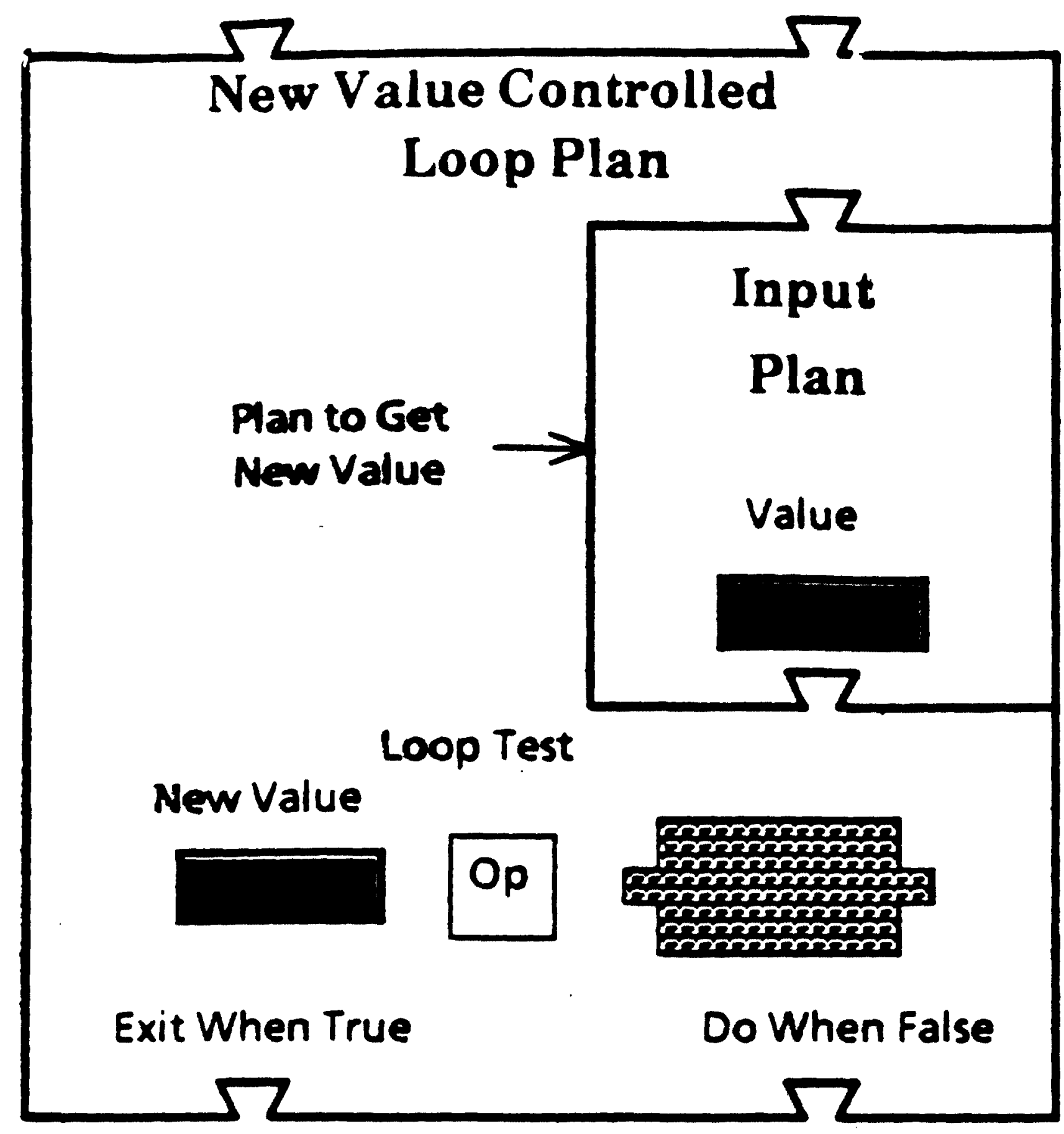

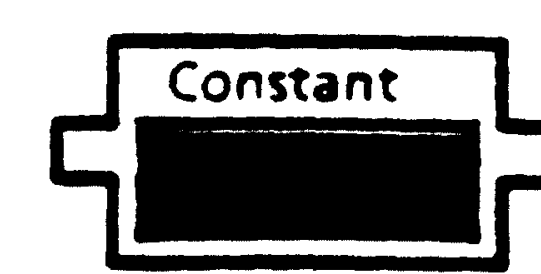

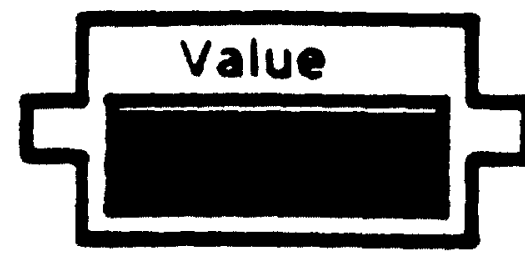

$\sqrt{2}$

Compute

Plan

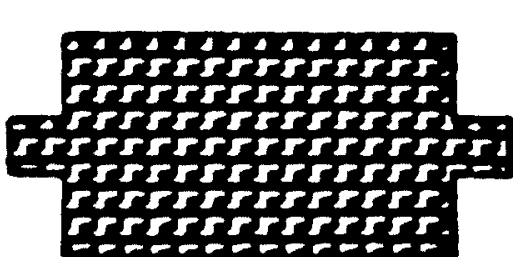

Op

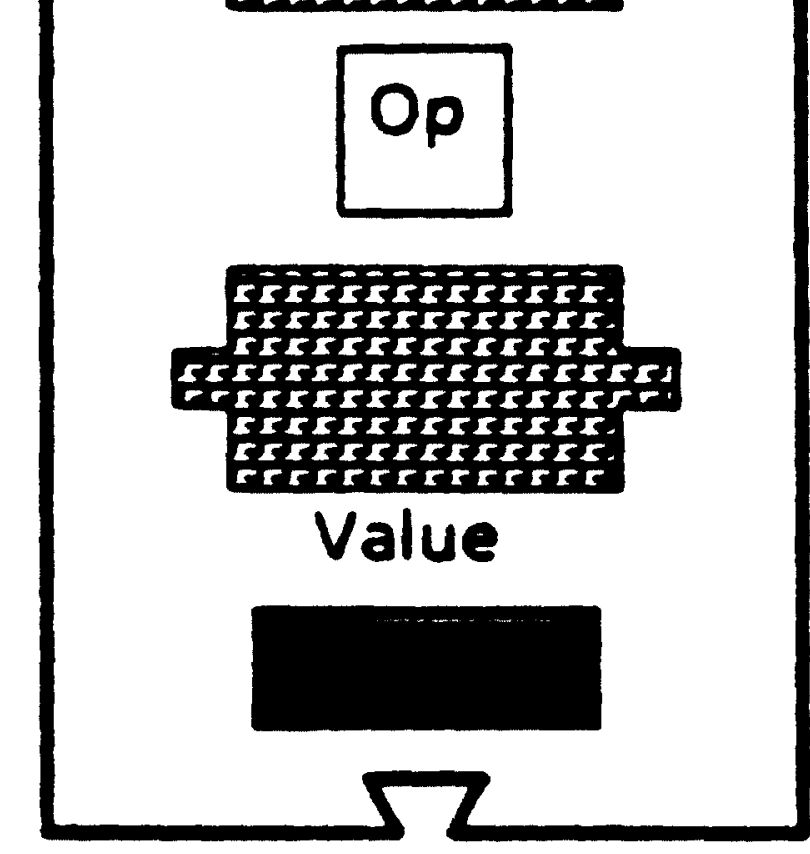

z

Running 'Total Plan Update

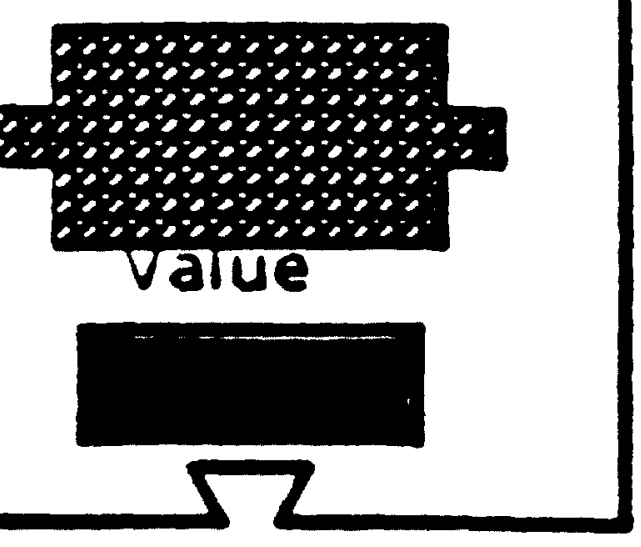

12

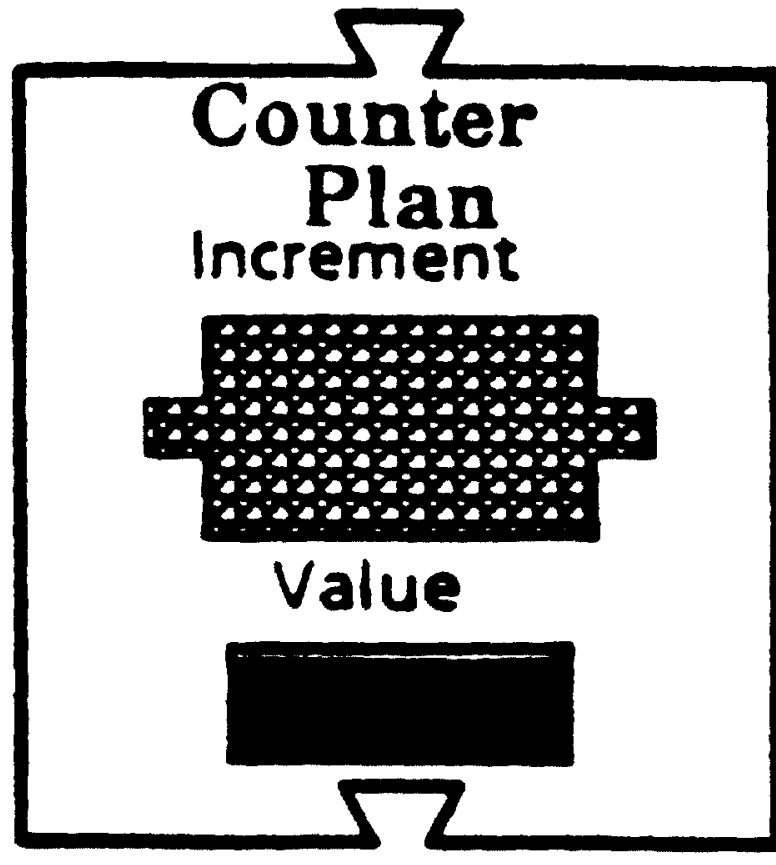

$\sqrt{2}$
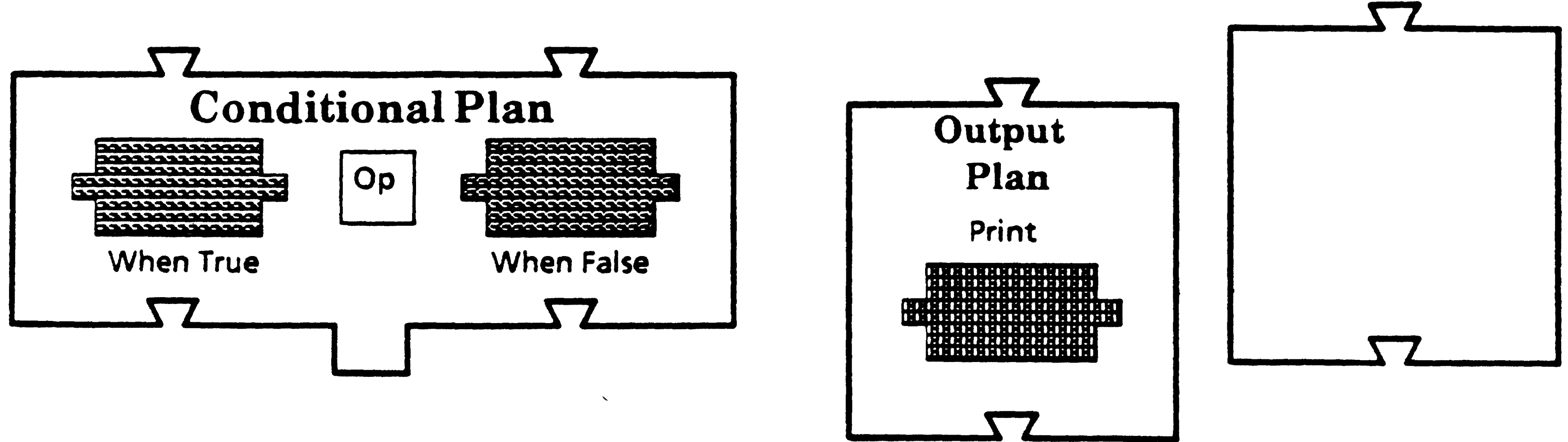


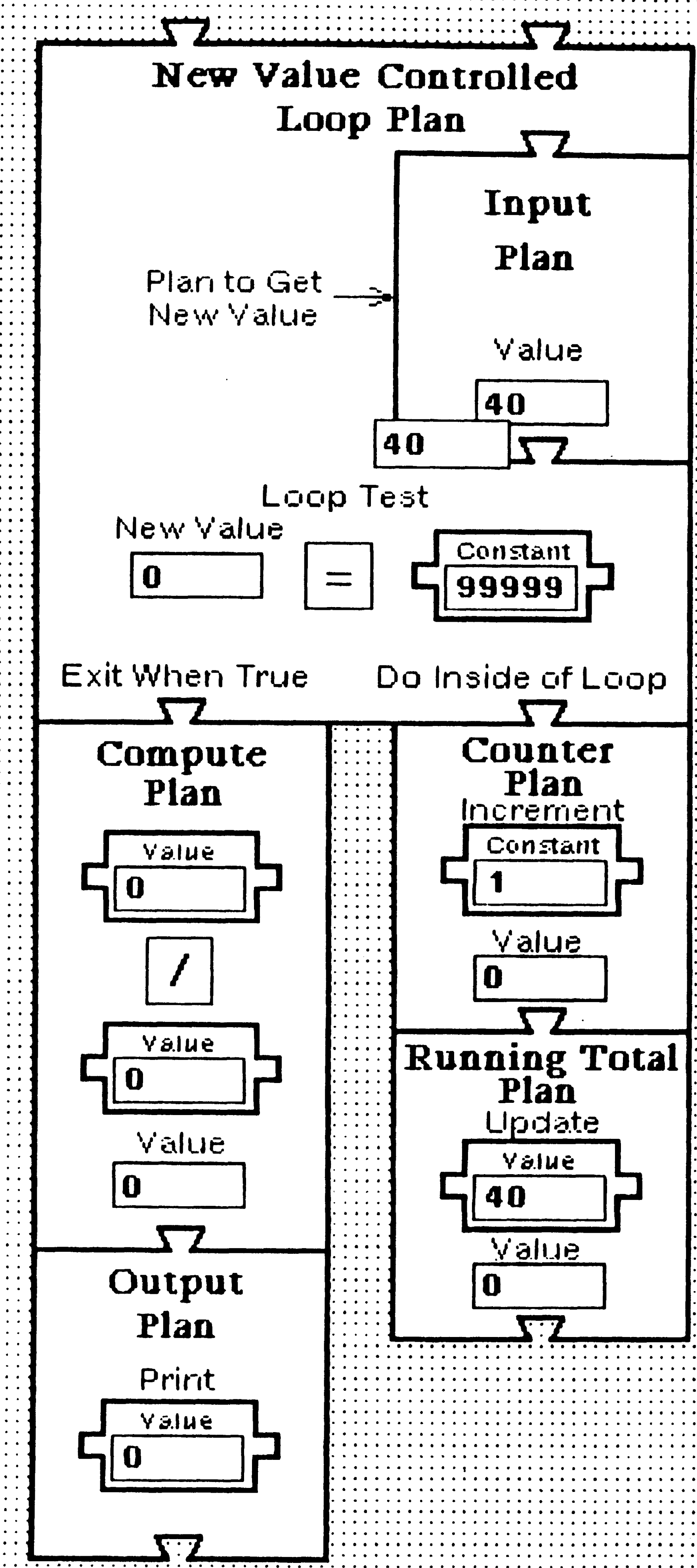

